\title{
THE ADELIC ZETA FUNCTION ASSOCIATED WITH THE SPACE OF BINARY CUBIC FORMS WITH COEFFICIENTS IN A FUNCTION FIELD
}

BORIS A. DATSKOVSKY

\begin{abstract}
In this paper we study the adelic zeta function associated with the prehomogeneous vector space of binary cubic forms, defined over a function field. We establish its rationality, find its poles and residues and a simple functional equation that this zeta function satisfies.
\end{abstract}

Introduction. The subject of integral equivalence of binary cubic forms, although not as popular or well developed as the theory of integral equivalence of binary quadratic forms, has received a fair amount of attention. The first paper on this topic [9], written by Eisenstein, appeared in 1844. Later on several other first-rate mathematicians tried their hand at the subject. Among others, the list includes Arndt, Cayley, Hermite, and Davenport. The classical method of attacking this problem is to draw upon a one-to-one discriminant-preserving relation between binary cubic and binary quadratic forms, which assigns to a cubic form its Hessian, and then use the reduction theory for binary quadratic forms to obtain results on class numbers of binary cubic forms. The best of the classical results are due to Davenport [5], who showed that

$$
\begin{aligned}
& \sum_{n \leqslant x} h^{(i r)}(n)=\pi^{2} / 36 x+O\left(x^{15 / 16}\right), \\
& \sum_{n \leqslant x} h^{(i r)}(-n)=\pi^{2} / 12 x+O\left(x^{15 / 16}\right),
\end{aligned}
$$

where $h^{(i r)}(n)$ is the class number of irreducible binary cubic forms of discriminant $n$.

The credit for a new surge of interest in the study of integral equivalence of binary cubic forms must indisputably go to Shintani, for it was his 1972 paper [25] that put this study on modern footing. In that paper Shintani introduced a certain zeta function which, as it turns out, decomposes into a linear combination of products of Dirichlet series, whose coefficients are "weighted" class numbers of binary cubic forms, and certain Mellin transforms. By studying analytic properties of the zeta function Shintani was able to improve the error term in Davenport's result to $O\left(x^{5 / 6}\right)$. It is of interest to note that the zeta function and the Dirichlet series it

Received by the editors September 3, 1985.

1980 Mathematics Subject Classification (1985 Revision). Primary 12A70: Secondary 10C10, 12A90.

(1987 American Mathematical Society $0002-9947 / 87 \$ 1.00+\$ .25$ per page 
engenders are one of the most interesting examples of zeta functions and Dirichlet series associated with prehomogeneous vector spaces. For the general theory of zeta functions associated with prehomogeneous vector spaces we refer the reader to a paper of Sato and Shintani [24].

Although the results Shintani obtains in [25] are only a slight improvement on the results of Davenport [5], his method has one enormous advantage: it can be carried over to the study of binary cubic forms with coefficients in a number field or in a function field. Moreover, an adelic version of the zeta function can be studied. Such "adelization" makes the treatment of the zeta function more elegant. Perhaps, another motivation for doing things adelicly is a generalization of results of Davenport and Heilbronn [7]. In that paper they observed a one-to-one discriminant-preserving relation between cubic extensions of $\mathbf{Q}$ and a certain class of irreducible integral binary cubic forms, given by congruence conditions at each prime $p$, and then used a filtering argument together with asymptotic formulas of [5] to obtain three beautiful results: a formula for the density of discriminants of cubic fields, an "inverse" Chebotarev density theorem for cubic fields, and a formula for the average size of 3-class-numbers of quadratic fields. After a great deal of effort, Wright [35] was able to generalize their method to a number field of class number 1. However, these results fall quite naturally, for any number field or function field of characteristic other than 2 or 3 , out of the study of the adelic zeta function.

The global theory of the adelic zeta function, defined over a number field, is treated quite elegantly in [36]. In the present paper, we deal with the function field case. Although there are many parallels between the two cases, some features, such as rationality of the zeta function, are peculiar to the function field situation alone. This is why we believe this case warrants special attention.

This paper is the first in a series of three papers. In the second paper (coauthored with Wright), we will present a treatment of the local theory of the zeta function associated with the space of binary cubic forms. Finally, in Part III of the series we will draw upon the results of Part II to deduce the class number and density of discriminants results a lá Davenport and Heilbronn [7].

The author is grateful to Professors Barry Mazur of Harvard and David Wright of MIT for getting him interested in the subject of binary cubic forms and for many useful and stimulating discussions in the course of preparation of this work.

Notations. Throughout this paper, $V$ will stand for the four-dimensional affine space and $G$ for the algebraic group $\mathrm{Gl}_{2}$. K will denote a function field in one variable over a finite field of constants $\mathbf{F}_{q}, q \neq 2^{n}$ or $3^{n}$, except in $\S 1$ where $K$ stands for an arbitrary field. A will denote the ring of adeles of $K, \mathbf{A}^{\times}$the idele group in $\mathbf{A}$, $|\cdot|_{\mathbf{A}}$ the adelic norm, and $\mathbf{A}^{n}$ the set of elements of $\mathbf{A}^{\times}$of norm $q^{n}$. The genus of $K$ will be denoted by $g$, not to be confused with $g$, used to denote an element of $\mathrm{Gl}_{2}$.

If $X$ is an algebraic variety, then for any ring $R$ we will write $X_{R}$ for the set of points of $X$ with coordinates in $R$. Hence the notations $V_{K}, V_{\mathbf{A}}, G_{K}$, and $G_{\mathbf{A}}$.

The following "abuse" of notation will be perpetrated: Let $\Gamma$ denote a discrete subgroup of $G$. Then we will write $d g$ both for a left-invariant measure on $G$ and the 
left-invariant measure on $G / \Gamma$ such that

$$
\int_{G} f(g) d g=\int_{G / \Gamma} \sum_{\gamma \in \Gamma} f(g \gamma) d g .
$$

A few peculiar notations that we use to denote elements of $G l_{2}$ are as follows: $k$ will stand for an element of the standard maximal compact subgroup of $G_{\mathbf{A}}$,

$$
\begin{aligned}
& a\left(t_{1}, t_{2}\right)=\left(\begin{array}{ll}
t_{1} & 0 \\
0 & t_{2}
\end{array}\right), \quad d(t)=\left(\begin{array}{ll}
t & 0 \\
0 & t
\end{array}\right), \\
& a(t)=\left(\begin{array}{ll}
1 & 0 \\
0 & t
\end{array}\right), \quad \text { and } \quad n(u)=\left(\begin{array}{ll}
1 & 0 \\
u & 1
\end{array}\right) .
\end{aligned}
$$

In the subsequent treatment of the adelic zeta function we adopt the language of quasicharacters. Let $\Omega$ denote the space of quasicharacters on $\mathbf{A}^{\times} / K^{\times}$. By choosing a uniformizer $\pi \in \mathbf{A},|\pi|_{\mathbf{A}}=q$, we induce a decomposition $\mathbf{A}^{\times} \cong \mathbf{A}^{0} \times \mathbf{Z}$. A quasicharacter $\omega \in \Omega$ then decomposes as $\omega=\tilde{\omega} \omega_{s}$, where

$$
\tilde{\omega}=\left\{\begin{array}{ll}
\omega & \text { on } \mathbf{A}^{0}, \\
1 & \text { on } \mathbf{Z},
\end{array} \text { and } \quad \omega_{s}(t)=|t|_{\mathbf{A}}^{s}\right.
$$

(for example, $\omega_{2}(t)=|t|_{\mathbf{A}}^{2}$ and $\omega_{-1 / 3}(t)=|t|_{\mathbf{A}}^{-1 / 3}$; both of these characteristics will occur in the discussion that follows). The notation $\omega=\tilde{\omega} \omega_{s}$ will be used consistently throughout this paper.

Another notation that occurs frequently in $\S 3$ is $\delta(\omega)$ :

$$
\delta(\omega)= \begin{cases}1 & \text { if } \tilde{\omega}=1 \\ 0 & \text { otherwise }\end{cases}
$$

Finally, $\mathbf{Z}$ denotes the ring of integers.

1. The space of binary cubic forms. Denote by $V$ the four-dimensional affine space. We identify $V$ with the space of binary cubic forms via the correspondence

$$
x=\left(x_{1}, x_{2}, x_{3}, x_{4}\right) \leftrightarrow F_{x}(u, v)=x_{1} u^{3}+x_{2} u^{2} v+x_{3} u v^{2}+x_{4} v^{3} .
$$

Define the action of the algebraic group $G=G l_{2}$ on $V$ by

$$
F_{g \circ x}(u, v)=\frac{1}{\operatorname{det}(g)} F_{x}((u, v) g)=\frac{1}{a d-b c} F_{x}(a u+c v, b u+d v),
$$

where $g=\left(\begin{array}{ll}a & b \\ c & d\end{array}\right) \in G l_{2}$. The action is contrived so that $\left(\begin{array}{ll}a & 0 \\ 0 & a\end{array}\right) \circ x=a x$.

The object of this section is to list some facts pertaining to this action. While some of the facts will not be used in this paper, they will, we hope, contribute to the reader's understanding of the geometry of the situation studied here.

We call a polynomial $Q(x), x=\left(x_{1}, x_{2}, x_{3}, x_{4}\right)$, a relative $G$-invariant if there exists a rational character $\chi$ on $G$ such that $Q(g \circ x)=\chi(g) Q(x)$ for any $g \in G$. The feature that makes this action particularly amenable to study is that the group of polynomial invariants in this case generated by a single polynomial

$$
P(x)=x_{2}^{2} x_{3}^{2}+18 x_{1} x_{2} x_{3} x_{4}-4 x_{2}^{3} x_{4}-4 x_{1} x_{3}^{3}-27 x_{1}^{2} x_{4}^{2} .
$$

The polynomial $P(x)$ satisfies

$$
P(g \circ x)=(\operatorname{det}(g))^{2} P(x) .
$$


A more satisfactory description of $P(x)$ can be given when working over a field $K$. The form $F_{x}(u, v), x \in V_{K}$, splits over the algebraic closure $\bar{K}$ of $K$ into a product of linear factors

$$
F_{x}(u, v)=\left(\alpha_{1} u+\beta_{1} v\right)\left(\alpha_{2} u+\beta_{2} v\right)\left(\alpha_{3} u+\beta_{3} v\right) .
$$

Then it is easy to verify that

$$
P(x)=\prod_{i<j}\left(\alpha_{i} \beta_{j}-\alpha_{j} \beta_{i}\right)^{2},
$$

the discriminant of $F_{x}$.

Our next task is to describe the orbital structure of the space $V_{K}$. Let $F_{x}$ split into a product of linear factors as above. We call the points $\lambda_{i}=\left[\alpha_{i}: \beta_{i}\right], i=1,2,3$, in the projective space $\mathbf{P}^{1}(\bar{K})$ the roots of $x$. We will say that a form $x$ is nonsingular if all its roots are distinct, and singular otherwise. The set of all nonsingular forms will be denoted by $V^{\prime}$, and the set of all singular forms by $S$. We note that $V^{\prime}=\{x \in$ $V \mid P(x) \neq 0\}$.

The roots of the form $g \circ x, g=\left(\begin{array}{ll}a & b \\ c & d\end{array}\right)$, are given by

$$
\mu_{i}=g\left(\lambda_{i}\right)=\left[a \alpha_{i}+b \beta_{i}: c \alpha_{i}+d \beta_{i}\right] \text {. }
$$

Since a linear fractional transformation can take any three points in $\mathbf{P}^{\mathrm{l}}(\bar{K})$ to any other three points and since we can adjust the leading coefficient of a cubic form via action by matrices of the form $\left(\begin{array}{cc}a & 0 \\ 0 & a\end{array}\right)$, two forms in $V_{\bar{K}}$ are $G_{\bar{K}^{-}}$-equivalent if and only if they have the same number of roots. Thus $V_{\bar{K}}^{\prime}$ is a single $G_{\bar{K}}$-orbit and $S_{\bar{K}}$ decomposes into three $G_{\bar{K}}$-orbits: the zero form, forms with a triple root, and forms with a double root.

Since over an algebraicly closed field the space $V$ contains a Zariski open $G$-orbit, the pair $(G, V)$ is a prehomogeneous vector space. For the general theory of zeta functions and Dirichlet series associated with prehomogeneous vector spaces we refer the reader to [24].

Returning to the $G_{K}$-orbit structure of $V_{K}$, let $K(x)$ denote the smallest extension of $K$ over which $F_{x}$ splits. Clearly $K(x)=K\left(\alpha_{1} / \beta_{1}, \alpha_{2} / \beta_{2}, \alpha_{3} / \beta_{3}\right)$. It is a rather simple exercise in Galois theory to show that two nonsingular forms $x$ and $x^{\prime}$ lie in the same $G_{K}$-orbit if and only if $K(x)=K\left(x^{\prime}\right)$. In particular, when $K$ is a complete field, $\operatorname{char}(K) \neq 2,3, V_{K}^{\prime}$ decomposes into finitely many $G_{K}$-orbits. This is another essential feature of the situation studied in [24].

We now turn to singular forms. Let $B_{K}$ denote the Borel subgroup of lower triangular matrices and $D_{K}$ the subgroup of diagonal matrices in $G_{K}$. We will need the following explicit description of the singular orbits.

PROPOSITION 1.1. The singular $G_{K^{-}}$orbits are

$$
\begin{aligned}
& S_{K, 0}=\{0\}, \\
& S_{K, 3}=\left\{x \in V_{K} \mid x \text { has a triple root }\right\}=\bigcup_{\gamma \in C_{K} / B_{K}} \bigcup_{a \in K^{\times}} \gamma \circ(0,0,0, a), \\
& S_{K, 2}=\left\{x \in V_{K} \mid x \text { has a double root }\right\}=\bigcup_{\gamma \in G_{K^{\prime}} D_{K}} \bigcup_{a \in K^{\times}} \gamma \circ(0,0, a, 0) .
\end{aligned}
$$


Proof. Since the splitting field of a singular form is $K$ itself, any two singular forms, having the same number of roots, are $G_{K}$-equivalent. The forms $(0,0,0, a)$ and $(0,0, a, 0)$ are forms with a triple and a double root respectively, and the stabilizers of the sets $\bigcup_{a \in K^{\times}}(0,0,0, a)$ and $\bigcup_{a \in K^{\times}}(0,0, a, 0)$ are respectively $B_{K}$ and $D_{K}$. Hence the decompositions of $S_{1}$ and $S_{2}$. Q.E.D.

More information about the space of binary cubic forms can be found in $\$ 2$ of [36] or in Chapter 1 of [4].

\section{The adelic zeta function: definition, convergence.}

2.1 From now on, $K$ will denote a function field in one variable over a finite field of constants $\mathbf{F}_{q}$, Char $(K) \neq 2$ or 3 . In this section we are going to define the adelic zeta function $Z(\omega, f)$, associated with the space of binary cubic forms, where $\omega$ is a quasicharacter on the group of ideles of $K$ trivial on $K^{\times}$, and $f$ is a Schwartz-Bruhat function on the space of adelic cubic forms $V_{\mathbf{A}}$. First, however, we have to set down the necessary notations.

Denote by $M$ the complete set of valuations of $K$. For each $\nu \in M$ let $K_{\nu}$ denote the completion of $K$ at $\nu, O_{\nu}$ the ring of integers in $K_{\nu}, O_{v}^{\times}$the group of units in $O_{\nu}$, and $|\cdot|_{\nu}$ the $\nu$-norm on $K_{\nu}$. Let $\mathbf{A}$ denote the ring of adeles and $\mathbf{A}^{\times}$the group of ideles of $K$. Both $\mathbf{A}$ and $\mathbf{A}^{\times}$are endowed with usual topologies which make them into a locally compact topological ring and group, respectively. The group $\mathbf{A}^{\times}$is also endowed with the adelic absolute value, denoted by $|\cdot|_{\mathbf{A}} ;|x|_{\mathbf{A}}=\prod_{\nu \in M}\left|x_{\nu}\right|_{\nu}$, $x=\left(x_{\nu}\right) \in \mathbf{A}^{\times}$.

The field $K$ naturally embeds into $A$ along the diagonal, and from now on we will identify $K$ with its image in A. With this identification $G_{K}$ becomes a discrete subgroup of $G_{\mathrm{A}}$ and $V_{K}$ a $G_{K}$-invariant lattice in $V_{\mathbf{A}}$.

Denote by $\mathscr{S}\left(V_{\mathbf{A}}\right)$ the space of locally constant functions on $V_{\mathrm{A}}$ with compact support and by $\Omega$ the space of quasicharacters on $\mathrm{A}^{\times}$that are trivial on $K^{\times}$. Then the adelic zeta function is defined by

$$
Z(\omega, f)=\int_{G_{A} / G_{K}} \omega(\operatorname{det}(g)) \sum_{x \in V_{K}^{\prime}} f(g \circ x) d g,
$$

where $\omega \in \Omega, f \in \mathscr{S}\left(V_{\mathbf{A}}\right)$, and $d g$ is a left-invariant Haar measure on $G_{\mathbf{A}}$ to be normalized in $\$ 2.3$.

2.2 A few words about the quasicharacter space $\Omega$ are in order here. Let $\mathbf{A}^{0}=\left\{\left.x \in \mathbf{A}^{\times}|| x\right|_{\mathbf{A}}=1\right\}$. Then $\mathbf{A}^{\times} / K^{\times} \cong \mathbf{A}^{0} / K^{\times} \times \mathbf{Z}$. This isomorphism is effected by choosing a uniformizer $\pi \in \mathbf{A}^{\times},|\pi|_{\mathbf{A}}=q$. Any quasicharacter $\omega \in \Omega$ can be written as $\omega=\tilde{\omega} \omega_{s}$, where $\tilde{\omega}$ is the quasicharacter that coincides with $\omega$ on $\mathbf{A}^{0}$ and is trivial on $\mathbf{Z}$, and $\omega_{s}$ is given by $\omega_{s}(x)=|x|_{\mathbf{A}}^{s}$. Since $\mathbf{A}^{0} / K^{\times}$is compact, $\tilde{\omega}$ is actually a character.

While both $\tilde{\omega}$ and $s$ depend on the choice of the uniformizer $\pi, \operatorname{Re}(s)$ does not. We define $\operatorname{Re}(\omega)=\operatorname{Re}(s), \omega=\tilde{\omega} \omega_{s}$. In $\S 2.5$ we are going to show that the integral defining $Z(\omega, f)$ converges absolutely in the region $\operatorname{Re}(\omega)>2$.

We say that a complex-valued function $\Phi(\omega)$ with domain in the space $\Omega$ is holomorphic, meromorphic, or rational if it is, respectively, a meromorphic, holomorphic, or rational function of $q^{s}, \omega=\tilde{\omega} \omega_{s}$. Our ultimate goal is to show that $Z(\omega, f)$ is a rational function of $\omega$. 
2.3 To establish a convergence result for $Z(\omega, f)$ we need an explicit description of the left-invariant Haar measure $d g$ on $G_{\mathbf{A}}$, a description for a fundamental domain of $G_{K}$ in $G_{\mathbf{A}}$, or of a slightly bigger set containing it, and a bound on the size of the integrand. We begin with the Haar measure.

Denote by $B, U$, and $D$ the subgroups of lower triangular matrices, unipotent matrices, and diagonal matrices in $G$, respectively. For each $\nu \in M$ put $k_{\nu}=G l_{2}\left(O_{\nu}\right)$, and set $k=\prod_{\nu \in M} k_{\nu}$. Then $k$ is the standard maximal compact subgroup of $G_{\mathbf{A}}$. We will use the following notation to denote individual matrices: $k$ will stand for an element of $k$,

$$
\begin{gathered}
n(u)=\left(\begin{array}{ll}
1 & 0 \\
u & 1
\end{array}\right), \quad a\left(t_{1}, t_{2}\right)=\left(\begin{array}{cc}
t_{1} & 0 \\
0 & t_{2}
\end{array}\right), \\
a(t)=a(1, t)=\left(\begin{array}{ll}
1 & 0 \\
0 & t
\end{array}\right), \quad \text { and } d(t)=a(t, t)=\left(\begin{array}{ll}
t & 0 \\
0 & t
\end{array}\right) .
\end{gathered}
$$

We note that $a(t) n(u)=n(t u) a(t)$.

By Iwasawa decomposition the map $k \times U_{\mathbf{A}} \times D_{\mathbf{A}} \rightarrow G_{\mathbf{A}}, k \times n(u) \times a\left(t_{1}, t_{2}\right) \rightarrow$ $k n(u) a\left(t_{1}, t_{2}\right)$, is onto. A left-invariant Haar measure $d g$ is then explicitly given by $d g=d k d u d^{\times} t_{1} d^{\times} t_{2}$, where $d k$ is a Haar measure on $k, d u$ is a Haar measure on $\mathbf{A}$, and $d^{\times} t_{i}, i=1,2$, is a Haar measure on $\mathbf{A}^{\times}$. We normalize $d k, d u$, and $d^{\times} t_{i}$, and thus $d g$, by settinig

$$
\int_{k} d k=1, \quad \int_{\mathbf{A} / K} d u=1, \quad \text { and } \int_{\mathbf{A}^{0} / K^{\times}} d^{\times} t_{i}=1
$$

For computational purposes we will use a slightly different version of the Iwasawa decomposition and the description of the measure $d g$ which goes with it. Write $g=k d\left(t_{1}\right) a(t) n(u)=k n(t u) a\left(t_{1}, t_{1} t\right)$. Then $d g=|t|_{\mathbf{A}} d k d u d^{\times} t_{1} d^{\times} t$.

Later on in the paper we will have to consider integrals over $G_{\mathbf{A}} / B_{K}$ and $G_{\mathbf{A}} / D_{k}$ with respect to the measure $d g$. Such integrals are explicitly given as follows:

$$
\begin{aligned}
& \int_{G_{\mathbf{A}} / B_{K}} \phi(g) d g=\int_{k} \int_{\mathbf{A}^{\times} / K^{\times}} \int_{\mathbf{A}^{\times} / K^{\times}} \int_{\mathbf{A} / K} \phi\left(k d\left(t_{1}\right) a(t) n(u)\right)|t|_{\mathbf{A}} d k d u d^{\times} t_{1} d^{\times} t, \\
& \int_{G_{\mathbf{A}} / D_{K}} \phi(g) d g=\int_{k} \int_{\mathbf{A}^{\times} / K^{\times}} \int_{\mathbf{A}^{\times} / K^{\times}} \int_{\mathbf{A}} \phi\left(k d\left(t_{1}\right) a(t) n(u)\right)|t|_{\mathbf{A}} d k d u d^{\times} t_{1} d^{\times} t,
\end{aligned}
$$

where $\phi(g)$ is a locally constant function on $G_{\mathbf{A}} / B_{K}$ (resp. on $G_{\mathbf{A}} / D_{K}$ ) with compact support.

The $t_{1}, t$, and $u$ of the Iwasawa decomposition $g=k d\left(t_{1}\right) a(t) n(u)$ are not unique; however, $t_{1}$ and $t$ are unique up to multiplication by elements of $\mathbf{A}^{\times}(\varnothing)=$ $\Pi_{\nu \in M} O_{\nu}^{\times}$(hence ord ${ }_{\nu} t(g)$, ord ${ }_{\nu} t_{1}(g),|t(g)|_{\mathbf{A}}$, and $\left|t_{1}(g)\right|_{\mathbf{A}}$ are well defined), and $u$ is uniquely determined modulo $t^{-1} \mathbf{A}(\varnothing), \mathbf{A}(\varnothing)=\prod_{\nu \in M} O_{\nu}$.

2.4 Our next task is to describe a fundamental domain of $G_{K}$ in $G_{\mathbf{A}}$. Set $\Theta=\left\{\left.g \in G_{\mathbf{A}}|| t(g)\right|_{\mathbf{A}}<q^{2 g}\right\}$, where $g$ is the genus of the field $K$. By a reduction theorem of Harder $[\mathbf{1 0}] \Theta G_{K}=G_{\mathbf{A}}$. 
The set $\Theta$ is invariant under multiplication by $B_{K}$ on the right. Hence a fundamental domain of $B_{K}$ in $\Theta$ contains a fundamental domain of $G_{K}$ in $G_{\mathbf{A}}$. An explicit description of the former is contained in the following lemma:

LEMMA 2.1. $\Theta=\cup_{u} \cup_{t_{1}, t} k d\left(t_{1}\right) a(t) n(u) B_{K}$, where $t_{1}$ and $t$ run over a set of representatives of $\mathbf{A}^{\times} / K^{\times} \mathbf{A}^{\times}(\varnothing)$ in $\mathbf{A}^{\times}$, subject to the condition $|t|_{\mathbf{A}} \leqslant q^{2 g}$, and $u$ runs over a finite set in $\mathbf{A}$.

Proof. The part of the lemma concerning $t_{1}$ and $t$ is trivial. As for the $u$ 's, $k d\left(t_{1}\right) a(t) n(u) B_{K}=k d\left(t_{1}\right) a(t) n\left(u^{\prime}\right) B_{K}$ provided $\left(u^{\prime}-u\right) \in t^{-1} \mathbf{A}(\varnothing)+K$. Therefore we can pick $u$ 's in a set of representatives of $\mathbf{A} /\left(t^{-1} \mathbf{A}(\varnothing)+K\right)$ in $\mathbf{A}$. We observe that

(i) For each $t \in \mathbf{A}^{\times}$, the set $\mathbf{A} /\left(t^{-1} \mathbf{A}(\varnothing)+K\right)$ is finite.

(ii) By the Riemann-Roch theorem, $\mathbf{A}=t^{-1} \mathbf{A}(\varnothing)+K$ whenever $|t|_{\mathbf{A}} \leqslant q^{1-2 g}$. Thus for all such $t$ we can take $u=0$.

(iii) Since $\mathbf{A}^{0} / K^{\times} \mathbf{A}^{\times}(\varnothing)$ is finite, the set $\left\{t \in \mathbf{A}^{\times} /\left.K^{\times} \mathbf{A}^{\times}(\varnothing)\left|q^{2-2 g} \leqslant\right| t\right|_{\mathbf{A}} \leqslant q^{2 g}\right\}$ is finite.

Combination of (i) through (iii) implies that the set of $u$ 's is actually finite. Q.E.D.

For reasons that will become apparent shortly it is convenient to interchange $a(t)$ and $n(u) . a(t) n(u)=n(t u) a(t)$, and since $u$ can be taken to be zero for all but finitely many $t$ 's in Lemma 2.1, the set of all $(t u)$ 's is still finite. Hence

LemMa 2.2. Every element in $\Theta$ is $B_{K}$-right-equivalent to an element of the set

$$
\mathscr{S}=\bigcup_{u} \bigcup_{t_{1}, t} k n(u) d\left(t_{1}\right) a(t),
$$

where $t_{1}$ and $t$ run over a set of representatives of $\mathbf{A}^{\times} / K^{\times} \mathbf{A}(\varnothing)$ in $\mathbf{A}^{\times},|t|_{\mathbf{A}} \leqslant q^{2 g}$, and $u$ runs over a finite set in $\mathbf{A}$.

The set $\mathscr{S}$ is a function field analogue of a Siegel domain.

2.5. It remains to establish a bound for the "mystery" factor, $\Sigma_{x \in V_{K}^{\prime}} f(g \circ x)$, in the integrand.

LEMMA 2.3. There exists an integer $N>0$ such that for any $g \in \Theta$

(i) $\sum_{x \in V_{K}^{\prime}}|f(g \circ x)|=0$ whenever $\left|t_{1}(g)\right|_{\mathbf{A}}>q^{N}$.

(ii) When $\left|t_{1}(g)\right| \leqslant q^{N}, \Sigma_{x \in V_{K}}|f(g \circ x)|=O\left(|t|_{\mathrm{A}}^{-3}\left|t_{1}\right|_{\mathrm{A}}^{-4}\right)$.

Proof. We can assume $g \in \mathscr{S}$. Furthermore, since $\cup_{u} k n(u), u$ being as in Lemma 2.2, is compact, we may assume $g=d\left(t_{1}\right) a(t)$.

Let $x=\left(x_{1}, x_{2}, x_{3}, x_{4}\right) \in V_{K}^{\prime}$. Suppose

$$
d\left(t_{1}\right) a(t) \circ x=\left(t_{1} t^{-1} x_{1}, t_{1} x_{2}, t_{1} t x_{3}, t_{1} t^{2} x_{4}\right) \in \operatorname{Supp}(f) .
$$

Since $\operatorname{Supp}(f)$ is compact, both the first and second coordinates of $d\left(t_{1}\right) a(t) \circ x$ must be bounded, that is there exists $N^{\prime}>0$ such that $\max \left(\left|t_{1} t^{-1} x_{1}\right|_{\mathbf{A}},\left|t_{1} x_{2}\right|_{\mathbf{A}}\right) \leqslant q^{N^{\prime}}$. But since $x$ is nonsingular, either $x_{1}$ or $x_{2} \neq 0, x_{1}, x_{2} \in K$, and therefore $\max \left(\left|x_{1}\right|_{\mathbf{A}},\left|x_{2}\right|_{\mathbf{A}}\right)=1$. Hence $\min \left(\left|t_{1} t^{-1}\right|_{\mathbf{A}},\left|t_{1}\right|_{\mathbf{A}}\right)=\left|t_{1}\right|_{\mathbf{A}} \min \left(\left|t^{-1}\right|_{\mathbf{A}}, 1\right) \leqslant q^{N^{\prime}}$. But for $g \in \Theta,|t|_{\mathbf{A}} \leqslant q^{2 g}$. Hence $\left|t_{1}\right|_{\mathbf{A}} \leqslant q^{N^{\prime}+2 g}$, which implies (i). 
To prove (ii) we can again assume that $g=d\left(t_{1}\right) a(t)$. We call $x \in V_{K} g$-allowable if $g \circ x \in \operatorname{Supp}(f)$, and $x_{i} \in K g$-allowable if it is the $i$ th coordinate of a $g$-allowable $x$. Since projections of $\operatorname{Supp}(f)$ on all four coordinates are compact sets, the numbers of $g$-allowable $x_{i}$ 's, $i=1,2,3,4$, are respectively $O\left(\max \left(\left|t_{1}^{-1}\right|_{\mathbf{A}}, 1\right)=\right.$ $O\left(\left|t_{1}\right|_{\mathbf{A}}^{-1}\right)$ (since $|t|_{\mathbf{A}}<q^{2 g}$ and $\left.\left|t_{1}\right|_{\mathbf{A}} \leqslant q^{N}\right), O\left(\left|t_{1}\right|_{\mathbf{A}}^{-1}\right), O\left(\left|t_{1} t\right|_{\mathbf{A}}^{-1}\right)$, and $O\left(\left|t_{1} t^{2}\right|_{\mathbf{A}}^{-1}\right)$. Therefore the number of $g$-allowable $x$ is $O\left(|t|_{A}^{-3}\left|t_{1}\right|_{\mathbf{A}}^{-4}\right)$, and since $|f(x)|$ is bounded from above, so is $\sum_{x \in v_{K}}|f(g \circ x)|$. Q.E.D.

2.6 We are now ready to prove

THEOREM 2.1. The integral that defines $Z(\omega, f)$ converges absolutely and locally uniformly in $\omega, \operatorname{Re}(\omega)>2$. In particular, $Z(\omega, f)$ is a holomorphic function of $\omega$ in the region $\operatorname{Re}(\omega)>2$.

Proof. Let $\operatorname{Re}(\omega)=\sigma$. Combining the results of $\S \S 2.3,2.4$, and 2.5 , we obtain

$$
\begin{aligned}
& \int_{G_{\mathbf{A}} / G_{K}}|\omega(\operatorname{det}(g))|\left|\sum_{x \in V_{K}^{\prime}} f(g \circ x)\right| d g \ll \int_{\Theta / B_{K}}|\operatorname{det}(g)|_{\mathbf{A}}^{\sigma} \sum_{x \in V_{K}^{\prime}}|f(g \circ x)| d g \\
& \left.\ll \int_{\mathbf{A}^{\times} / K^{\times}} \int_{\mathbf{A}^{\times} / K^{\times}}\left|t_{1}^{2} t\right|_{\mathbf{A}}^{\sigma}|t|_{\mathbf{A}}^{-3}\left|t_{1}\right|_{\mathbf{A}}^{-4}|| t\right|_{\mathbf{A}} d^{\times} t d^{\times} t_{1} \\
& \left|t_{1}\right|_{\mathbf{A}} \leqslant q^{N}|t|_{\mathbf{A}} \leqslant q^{2 g} \\
& =\left(\int_{\substack{\mathbf{A}^{\times} / K^{\times} \\
|t|_{\mathbf{A}} \leqslant q^{2 g}}}|t|_{\mathbf{A}}^{\sigma-2} d^{\times} t\right)\left(\int_{\substack{\mathbf{A}^{\times} / K^{\times} \\
\left|t_{1}\right|_{\mathbf{A}} \leqslant q^{N}}}\left|t_{1}\right|_{\mathbf{A}}^{2 \sigma-4} d^{\times} t_{1}\right)
\end{aligned}
$$

and each of the latter two integers converges absolutely and locally uniformly in $\sigma$, provided $\sigma>2$. Q.E.D.

The result of Theorem 2.1 is the best convergence result possible. In $\S 3$ we are going to show that $Z(\omega, f)$ has a pole at $\omega=\omega_{2}$.

2.7 We conclude this section with a little proposition which will come in quite handy in $\$ 3$ when we address the problem of analytic continuation of the zeta function. Set

$$
\lambda_{-}(g)=\left\{\begin{array}{ll}
1, & |\operatorname{det}(g)|_{\mathbf{A}}<1, \\
\frac{1}{2}, & |\operatorname{det}(g)|_{\mathbf{A}}=1, \\
0, & |\operatorname{det}(g)|_{\mathbf{A}}>1,
\end{array} \quad \lambda_{+}(g)= \begin{cases}0, & |\operatorname{det}(g)|_{\mathbf{A}}<1 \\
\frac{1}{2}, & |\operatorname{det}(g)|_{\mathbf{A}}=1 \\
1, & |\operatorname{det}(g)|_{\mathbf{A}}>1\end{cases}\right.
$$

and define

$$
\begin{aligned}
& Z_{-}(\omega, f)=\int_{G_{\mathbf{A}} / G_{K}} \omega(\operatorname{det}(g)) \lambda_{-}(g) \sum_{x \in V_{K}^{\prime}} f(g \circ x) d g, \\
& Z_{+}(\omega, f)=\int_{G_{\mathbf{A}} / G_{K}} \omega(\operatorname{det}(g)) \lambda_{+}(g) \sum_{x \in V_{K}^{\prime}} f(g \circ x) d g .
\end{aligned}
$$

Then we have

Proposition 2.1. $Z_{+}(\omega, f)$ is an entire function of $\omega$; moreover, it is a polynomial in $q^{s}, \omega=\tilde{\omega} \omega_{s}$. 
Proof. Let $G_{\mathbf{A}}^{+}=\left\{\left.g \in G_{\mathbf{A}}|| \operatorname{det}(g)\right|_{\mathbf{A}} \geqslant 1\right\}$ and $\mathscr{S}^{+}=\left\{\left.g \in \mathscr{S}|| \operatorname{det}(g)\right|_{\mathbf{A}} \geqslant 1\right\}$, where $\mathscr{S}$ is the set of Lemma 2.2. Then $\mathscr{S}^{+}$contains a fundamental domain of $G_{K}$ in $G_{\mathbf{A}}^{+}$. Now set $\mathscr{S}^{+}(N)=\left\{\left.g \in \mathscr{S}^{+}|| t_{1}(g)\right|_{\mathbf{A}} \leqslant q^{N}\right\}$. Then by Lemma 2.3(i), the integral that defines $Z_{+}(\omega, f)$ is actually an integral over a subset of $\mathscr{S}^{+}(N)$.

We claim that $\mathscr{S}^{+}(N)$ is compact. Indeed, $\mathscr{S}^{+}(N)=\bigcup_{u} \bigcup_{t_{1}, t} k n(u) d\left(t_{1}\right) a(t)$, where the number of $u$ 's is finite, $\left|t_{1}\right|_{\mathrm{A}} \leqslant q^{N},|t|_{\mathrm{A}} \leqslant q^{2 g},\left|t_{1}^{2} t\right|_{\mathrm{A}} \geqslant 1$, and $t_{1}$ and $t$ run over a set of representatives of $\mathbf{A}^{\times} / K^{\times} \mathbf{A}^{\times}(\varnothing)$ in $\mathbf{A}^{\times}$. Since $\left|t_{1}^{2} t\right|_{\mathbf{A}} \geqslant 1,\left|t_{1}\right|_{\mathbf{A}}^{2} \geqslant$ $|t|_{\mathbf{A}}^{-1} \geqslant q^{-2 g}$. Hence $q^{-g} \leqslant\left|t_{1}\right|_{\mathbf{A}} \leqslant q^{N}$. Similarly $q^{-2 N} \leqslant|t|_{\mathbf{A}} \leqslant q^{2 g}$. The number of such $t$ 's and $t_{1}$ 's is finite. Hence the claim.

Compactness of $\mathscr{S}^{+}(N)$ immediately implies the proposition. Q.E.D.

\section{The zeta function: analytic continuation and the functional equation.}

3.1 In this section we will address the problem of analytically continuing the zeta function beyond the region $\operatorname{Re}(\omega)>2$. In the process of analytic continuation we will show that $Z(\omega, f)$ is a rational function of $\omega$ and obtain an explicit formula for its singular part. Finally, we will show that $Z(\omega, f)$ satisfies a simple functional equation. The methods that we employ in this section are due to Shintani [25].

3.2 When it comes to analytic continuation of zeta functions, the Poisson summation formula provides an invaluable tool. Hence our next task is to describe the dual space $V_{\mathbf{A}}^{*}$ of $V_{\mathbf{A}}$.

Let $\psi$ be an additive character on $\mathbf{A}$ trivial on $K$. We identify $\mathbf{A}^{*}$ with $\mathbf{A}$ via the pairing $(a, b)=\psi(a b), a, b \in \mathbf{A}$. Under this identification, the lattice $K \in \mathbf{A}$ becomes self-dual.

Next, let $\langle$,$\rangle be a nondegenerate alternate bilinear form on V_{\mathbf{A}}$ given by

$$
\langle x, y\rangle=x_{1} y_{4}-\frac{1}{3} x_{2} y_{3}+\frac{1}{3} x_{3} y_{2}-x_{4} y_{1} \text {. }
$$

We identify $V_{\mathbf{A}}$ with its dual via the pairing $(x, y)=\psi(\langle x, y\rangle)$. Under this identification, $V_{K}^{*}=V_{K}$.

The choice of the form $\langle$,$\rangle here is far from arbitrary: for any g \in G_{\mathbf{A}},\langle x, y\rangle=$ $\left\langle g \circ x, g^{\prime} \circ y\right\rangle$ where $g^{\prime}=g / \operatorname{det}(g)$.

Set $d x=d x_{1} d x_{2} d x_{3} d x_{4}, x=\left(x_{1}, x_{2}, x_{3}, x_{4}\right)$, where each $d x_{i}$ is normalized by $\int_{\mathbf{A} / K} d x_{i}=1$. For $f \in \mathscr{S}\left(V_{\mathbf{A}}\right)$ define the Fourier transform $\hat{f}$ of $f$ by setting

$$
\hat{f}(y)=\int_{V_{\mathrm{A}}} f(x) \psi(\langle x, y\rangle) d x .
$$

Then the Poisson summation formula asserts that for any $f \in \mathscr{S}\left(V_{\mathrm{A}}\right)$

$$
\sum_{x \in L} f(x)=\frac{1}{v(L)} \sum_{x \in L^{*}} \hat{f}(x)
$$

where $L$ is any $K$-lattice in $V_{\mathbf{A}}, L^{*}$ is its dual lattice, and $v(L)$ is the volume of a fundamental domain of $L$ in $V_{\mathrm{A}}$. In particular,

$$
\sum_{x \in V_{K}} f(x)=\sum_{x \in V_{K}} \hat{f}(x) \text {. }
$$

Define an action of $G_{\mathbf{A}}$ on $\mathscr{S}\left(V_{\mathbf{A}}\right)$ by setting $(g \circ f)(x)=f\left(g^{-1} \circ x\right)$. The measure $d x$ satisfies $d(g \circ x)=|\operatorname{det}(g)|_{\mathbf{A}}^{2} d x$, and an easy computation now shows that

$$
(\widehat{g \circ f})=|\operatorname{det}(g)|_{\mathbf{A}}^{2}\left(g^{\prime} \circ \hat{f}\right)=\omega_{2}(\operatorname{det}(g))\left(g^{\prime} \circ \hat{f}\right) \text {. }
$$


Applying the Poisson summation formula to $\left(g^{-1} \circ f\right)$, we obtain

$$
\sum_{x \in V_{K}} f(g \circ x)=\omega_{-2}(\operatorname{det}(g)) \sum_{x \in V_{K}} \hat{f}\left(g^{\prime} \circ x\right),
$$

and

$$
\begin{aligned}
\sum_{x \in V_{K}^{\prime}} f(g \circ x)= & \omega_{-2}(\operatorname{det}(g)) \sum_{x \in V_{K}^{\prime}} \hat{f}\left(g^{\prime} \circ x\right) \\
& +\sum_{x \in S_{K}}\left(\omega_{-2}(\operatorname{det}(g)) \hat{f}\left(g^{\prime} \circ x\right)-f(g \circ x)\right) .
\end{aligned}
$$

The zeta function $Z(\omega, f)=Z_{-}(\omega, f)+Z_{+}(\omega, f)$. Then (3.1), applied to $Z_{-}$, followed by a change of variables $g \rightarrow g^{\prime}$, yields

Proposition 3.1. $Z(\omega, f)=Z_{+}(\omega, f)+Z_{+}\left(\omega_{2} \omega^{-1}, \hat{f}\right)+I(\omega, f)$, where

$$
I(\omega, f)=\int_{G_{\mathrm{A}} / G_{K}} \omega(\operatorname{det}(g)) \lambda_{-}(g) \sum_{x \in S_{K}}\left(\omega_{-2}(\operatorname{det}(g)) \hat{f}\left(g^{\prime} \circ x\right)-f(g \circ x)\right) d g .
$$

We know from $\S 2$ that $Z_{+}(\omega, f)$ and $Z_{+}\left(\omega_{2} \omega^{-1}, \hat{f}\right)$ are entire functions of $\omega$, and the integral that defines $I(\omega, f)$ converges absolutely and locally uniformly in $\omega$, $\operatorname{Re}(\omega)>2$, for it is a linear combination of integrals that are absolutely and locally uniformly convergent in the region $\operatorname{Re}(\omega)>2$. Thus an analytic continuation for $Z(\omega, f)$ hinges upon an analytic continuation of $I(\omega, f)$, and most of the work to come will be geared toward evaluating $I(\omega, f)$ explicitly.

3.3 The key to the integral $I(\omega, f)$ is a family of somewhat simpler integrals $I^{n}(\omega, f), n \in \mathbf{Z}$;

$$
I^{n}(\omega, f)=\int_{G_{\mathrm{A}}^{n} / G_{K}} \omega(\operatorname{det}(g))\left(\omega_{-2}(\operatorname{det}(g)) \sum_{x \in S_{K}} \hat{f}\left(g^{\prime} \circ x\right)-\sum_{x \in S_{K}} f(g \circ x)\right) d g,
$$

where $G_{\mathbf{A}}^{n}=\left\{\left.g \in G_{A}|| \operatorname{det}(g)\right|_{\mathbf{A}}=q^{n}\right\}$.

The set $S_{K}$ decomposes as $S_{K}=S_{K, 0} \cup S_{K, 1} \cup S_{K, 2}$, where $S_{K, 0}, S_{K, 1}$, and $S_{K, 2}$ are the three singular orbits of Proposition 1.1. Each singular orbit has a rather simple $G_{K}$-structure, and therefore we would like to evaluate each of the integrals

$$
\int_{G_{\mathbf{A}}^{\prime \prime} / G_{K}} \omega(\operatorname{det}(g))\left(\omega_{-2}(\operatorname{det}(g)) \sum_{x \in S_{K, i}} \hat{f}\left(g^{\prime} \circ x\right)-\sum_{x \in S_{K, i}} f(g \circ x)\right) d g,
$$

$i=0,1,2$, separately. To take matters further, we would like to consider just the integrals

$$
\int_{G_{A}^{n} / G_{K}} \omega(\operatorname{det}(g)) \sum_{x \in S_{K, l}} f(g \circ x) d g, \quad i=1,2,3 .
$$

This, however, cannot be done without introducing a suitable fudge factor. The next lemma will explain what sort of factor makes the integrals (3.2) convergent.

Let $\Theta$ be the set of $\$ 2.4$ and let $\Theta^{n}=\left\{\left.g \in \Theta|| \operatorname{det}(g)\right|_{\mathbf{A}}=q^{n}\right\}$. Denote by $L\left(\Theta^{n} / B_{K}, r\right)$ the set of all complex-valued locally constant functions $\phi(g)$ on $G_{\mathbf{A}} / B_{K}$ such that

$$
\int_{\Theta^{n} / B_{K}}|\phi(g)||t(g)|_{\mathbf{A}}^{r} d g<\infty
$$


For any $f \in \mathscr{S}\left(V_{\mathbf{A}}\right)$ define the function $J f: G_{\mathbf{A}} \rightarrow \mathbf{C}$ by setting $(J f)(g)=$ $\sum_{x \in S_{K}} f(g \circ x)$. Then we have

Lemma 3.1. For any $f \in \mathscr{S}\left(V_{\mathbf{A}}\right), J f \in L\left(\Theta^{n} / B_{K}, r\right)$ for any $r>1$.

Proof. To prove Lemma 3.1 we need an estimate on the size of $(J f)(g)$ analogous to the estimate of Lemma 2.3 (ii). The result of Lemma 2.3 no longer applies since $\left|t_{1}^{2}(g) t(g)\right|_{\mathbf{A}}=q^{n}$ for $g \in \Theta^{n}$, and therefore $\left|t_{1}(g)\right|_{\mathbf{A}}=q^{n / 2}|t(g)|_{\mathbf{A}}^{-1 / 2}$ is rather large. However, a slightly modified argument of Lemma 2.3(ii) shows that for $g \in \Theta^{n}, \Sigma_{n \in V_{K}}|f(g \circ x)|$, and therefore $|(J f)(g)|$, is $O\left(|t(g)|_{\mathbf{A}}^{-2}\right)$. Then

$$
\begin{gathered}
\int_{\Theta^{n} / B_{K}}|(J f)(g)||t(g)|_{\mathbf{A}}^{r} d g=\int_{k} \int_{\substack{\mathbf{A}^{\times} / K^{\times} \\
\left|t_{1}^{2} t\right|_{\mathbf{A}}=q^{n} \\
|t|_{\mathbf{A}} \leqslant q^{2 g}}} \int_{\mathbf{A}^{\times} / K^{\times}} \int_{\mathbf{A} / K}|t|_{\mathbf{A}}^{r-2}|t|_{\mathbf{A}} d k d u d^{\times} t d^{\times} t_{1} \\
\ll \int_{\substack{\mathbf{A}^{\times} / K^{\times} \\
|t|_{\mathbf{A}} \leqslant q^{2 g}}}|t|_{\mathbf{A}}^{r-1} d^{\times} t<\infty,
\end{gathered}
$$

provided $r>1$. Q.E.D.

Since a fundamental domain of $B_{K}$ in $\Theta^{n}$ contains a fundamental domain of $G_{K}$ in $G_{\mathbf{A}}$, a fudge factor that would make the integrals (3.2) converge ought to be $O\left(|t(g)|_{\mathrm{A}}^{1+\varepsilon}\right)$ on $\Theta^{n}$. It was Shintani's brilliant idea (see [25]) to introduce a "smoothed" Eisenstein series (originally due to Langlands [17]) as a fudge factor in (3.2). This series satisfies the 0 -condition on $\Theta^{n}$; moreover, it makes a particularly pleasant fudge factor because of its invariance properties under the action of certain subgroups of $G_{\mathrm{A}}$ and because it is so easy to recover the original integral from the "fudged up" one. We will use the method of Shintani to evaluate the integrals $I^{n}(\omega, f)$. It is of interest to note that this method is not restricted to the space of binary cubic forms: in a recent paper [4], Cogdell used Shintani's method to study congruence zeta functions for the quarternion algebra $M_{2}(\mathbf{Q})$.

3.4 We now give an outline of the properties of Eisenstein series that we will need. The main reference for the results quoted in this section is a paper of Li [20]. Other useful references on Eisenstein series are [16, 18, 19, and 34].

Let $\lambda_{1}, \lambda_{2}$ be quasicharacters on $\mathbf{A}^{\times} / K^{\times} \mathbf{A}^{\times}(\varnothing)$. Following $\mathrm{Li}$, we define the Eisenstein series $E\left(\cdot, \lambda_{1}, \lambda_{2}\right): G_{\mathbf{A}} \rightarrow \mathbf{C}$ by

$$
E\left(g, \lambda_{1}, \lambda_{2}\right)=\sum_{\gamma \in G_{K} / B_{K}} \lambda_{1}\left(t_{1}(g \gamma)\right) \lambda_{2}\left(t_{2}(g \gamma)\right)\left|t_{1}(g \gamma) / t_{2}(g \gamma)\right|_{\mathbf{A}}^{-1 / 2} .
$$

We note that $E\left(g, \lambda_{1}, \lambda_{2}\right)$ is well defined, provided the sum in (3.3) converges absolutely, and is invariant under multiplication by $k$ on the left and $G_{K}$ on the right.

The Eisenstein series satisfy a whole gamut of properties that one would expect Eisenstein series to satisfy. The theorem below summarizes the results of Theorems $2.3,3.2$, and 5.2 of $[20]$. 
THEOREM 3.1. (i) The Eisenstein series $E\left(\cdot, \lambda_{1}, \lambda_{2}\right)$ is absolutely and locally uniformly convergent for all $\lambda_{1}, \lambda_{2}$ with $\operatorname{Re}\left(\lambda_{1} \lambda_{2}^{-1}\right)>1$.

(ii) Let $\lambda_{1}=\lambda_{1} \omega_{s_{1}}$ and $\lambda_{2}=\lambda_{2} \omega_{s_{2}}$. Then for a fixed $g \in G_{\mathbf{A}}$,

$$
E\left(g, \lambda_{1}, \lambda_{2}\right)=P\left(g, \lambda_{1}, \lambda_{2}\right) / Q\left(\lambda_{1}^{-1} \lambda_{2}\right),
$$

where $P\left(g, \lambda_{1}, \lambda_{2}\right)$ is a polynomial in $q^{s_{1}}, q^{s_{2}}, q^{-s_{1}}$, and $q^{-s_{2}}$, and $Q\left(\lambda_{1}^{-1} \lambda_{2}\right)$ is a polynomial in $q^{s_{2}-s_{1}}$ independent of $g$.

(iii) The Eisenstein series $E\left(g, \lambda_{1}, \lambda_{2}\right)$ satisfies a functional equation

$$
E\left(g, \lambda_{1}, \lambda_{2}\right)=C\left(\lambda_{1}^{-1} \lambda_{2}\right) E\left(g, \lambda_{2}, \lambda_{1}\right)
$$

where

$$
C(\lambda)=\int_{\mathbf{A}} \lambda\left(\left(\begin{array}{ll}
u & 1 \\
1 & 0
\end{array}\right)\right)\left\|t\left(\left(\begin{array}{ll}
u & 1 \\
1 & 0
\end{array}\right)\right)\right\|_{\mathbf{A}} d u .
$$

$C(\lambda)$ is computed explicitly in Theorem 3.1 of [20].

For our purposes, we need not consider a general Eisenstein series; the Eisenstein series that we will use to construct a fudge factor is the series

$$
E(g, s)=E\left(g, \omega_{s / 2}, \omega_{-s / 2}\right)=\sum_{\gamma \in G_{K} / B_{K}}|t(g \gamma)|_{\mathbf{A}}^{-s / 2-1 / 2}
$$

This series converges in the half-plane $\operatorname{Re}(s)>1$ and is, in fact, a rational function of $q^{s}$.

Write $g \in G_{\mathrm{A}}$ as $g=k d\left(t_{1}\right) a(t) n(u)$. When $t$ is fixed, $E(g, s)$ becomes a function of $u$ which we denote by $E_{t}(u, S)$. Since $E(g, s)$ is a right $G_{K}$-invariant, $E_{t}(u, s)=E_{t}(u+a, s)$ for any $a \in K$. Hence it has a Fourier expansion:

$$
E_{t}(u, s)=\sum_{a \in K} C_{a}(t, s) \psi(a u)
$$

The Fourier expansion provides a valuable tool for the study of Eisenstein series because all the coefficients in (3.4) are immensely computable. To begin with, $E(g, s)$ is a left $k$-invariant; therefore $E_{t}(u, s)=E_{t}\left(u+u^{\prime}, s\right)$ for any $u^{\prime} \in \mathbf{A}(\phi) / t$, and hence all but finitely many $C_{a}(t, s)$ are zero. More precisely, we have

Proposition 3.2. Let $g=k d\left(t_{1}\right) a(t) n(u) \in G_{\mathbf{A}}$, and let

$$
E(g, s)=\sum_{a \in K} C_{a}(t, s) \psi(a u)
$$

be the Fourier expansion of $E(\cdot, s)$. Then

(i) $C_{0}(t, s)=|t|_{\mathbf{A}}^{-s / 2-1 / 2}+|t|_{\mathbf{A}}^{s / 2-1 / 2} q^{1-g \zeta_{K}}(s) / \zeta_{K}(s+1)$, where $\zeta_{K}(s)$ is the Dedekind zeta function of the field $K$.

(ii) Let $[t]=\sum_{\nu \in M}\left(\operatorname{ord}_{\nu}(t)\right) \nu$ denote the divisor of $t$ and let $[\psi]$ stand for the canonical divisor, associated with the character $\psi$. Then $C_{a}(t, s)=0$ for all $a \notin$ $L([\psi]-[t])$, the linear system of the divisor $[\psi]-[t]$. If $a \in L([\psi]-[t])$, then

$$
C_{a}(t, s)=|t|_{\mathrm{A}}^{s / 2-1 / 2} \frac{P_{a}(t, s)}{\zeta_{K}(s+1)},
$$

where $P_{a}(t, s)$ is a polynomial in $q^{s}$. In particular, $C_{a}(t, s)$ is a holomorphic function of $q^{s}$ in the half-plane $\operatorname{Re}(s)>-\frac{1}{2}$. 
Part (i) of Proposition 3.2 is a particular case of Theorem 3.1 of [20]; the same technique yields part (ii).

As evident from the Fourier expansion, $E(g, s)$ is rather large when $|t(g)|_{\mathbf{A}}$ is small and thus is unsuitable as a fudge factor. To produce a suitable fudge factor we introduce the following smoothing procedure: Let $\phi$ be an entire function such that for any $N>0$ and any $c_{1}, c_{2} \in \mathbf{R}$,

$$
\sup _{c_{2}<\operatorname{Re}(w)<c_{1}}\left(1+|w|^{N}\right)|\phi(w)|<\infty .
$$

Following [25], define the "smoothed" Eisenstein series $\mathscr{E}(g, \phi, w)$ by setting

$$
\mathscr{E}(g, \phi, w)=\frac{1}{2 \pi i} \int_{1<\operatorname{Re}(s)=x_{0}<\operatorname{Re}(w)} \frac{E(g, s)}{w-s} \phi(s) d s .
$$

The properties that make $\mathscr{E}(g, \phi, w)$ such a wonderful fudge factor are listed in the following lemma.

LEMMA 3.2. (i) $\mathscr{E}(g, \phi, w)$ is an analytic function of $w$ in the half-plane $\operatorname{Re}(w)>1$.

(ii) For $g \in \Theta, \mathscr{E}(g, \phi, w)=O\left(|t(g)|_{\mathrm{A}}^{(\operatorname{Re}(w)-1) / 2}\right)$.

(iii) $\lim _{w \rightarrow 1}\left(1-q^{1-w}\right) \mathscr{E}(g, \phi, w)=\phi(1) q^{1-g} \operatorname{Res}_{q} \zeta_{K} / \zeta_{K}(2)$, where $\operatorname{Res}_{q} \zeta_{K}=$ $\lim _{w \rightarrow 1}\left(1-q^{1-w}\right) \zeta_{K}(w)$; the limit converges uniformly in $g, g \in \Theta$.

(iv) For any function $f \in L_{1}\left(G_{\mathbf{A}} / G_{K}\right)$,

$$
\lim _{w \rightarrow 1}\left(1-q^{1-w}\right) \int_{G_{A} / G_{K}} f(g) \mathscr{E}(g, \phi, w) d g=\phi(1) q^{1-g} \frac{\operatorname{Res}_{q} \zeta_{K}}{\zeta_{K}(2)} \int_{G_{A} / G_{K}} f(g) d g .
$$

Proof. (i) follows immediately from Theorem 3.1(i).

(ii) By Proposition 3.2(ii), $C_{a}(t, s)=0$ for any $a \in K^{\times}$provided $|t|_{\mathrm{A}} \leqslant q^{1-2 g}$. Let $Z_{\mathbf{A}}=\left\{\left(\begin{array}{cc}t & 0 \\ 0 & t\end{array}\right) \mid t \in \mathbf{A}^{\times}\right\}$be the center of the group $G_{\mathbf{A}}$. Since, by Lemma 2.2, $Z_{\mathbf{A}} \backslash$ $\Theta / B_{K}$ is the union of the set $\left\{g \in Z_{\mathrm{A}} \backslash \Theta /\left.B_{K}|| t(g)\right|_{\mathrm{A}} \leqslant q^{1-2 g}\right\}$ and a compact set, it is enough to show that

$$
\frac{1}{2 \pi i} \int_{\operatorname{Re}(s)=x_{0}} \frac{C_{0}(t, s)}{w-s} \phi(s) d s=O\left(|t|_{\mathrm{A}}^{(\operatorname{Re}(w)-1) / 2}\right), \quad|t|_{\mathrm{A}} \leqslant q^{1-2 g} .
$$

By Proposition 3.2(ii),

$$
\begin{aligned}
\frac{1}{2 \pi i} \int_{\operatorname{Re}(s)=x_{0}} \frac{C_{0}(t, s)}{w-s} \phi(s) d s & =\frac{1}{2 \pi i} \int_{\operatorname{Re}(s)=x_{0}} \frac{|t|_{\mathbf{A}}^{-s / 2-1 / 2}}{w-s} \phi(s) d s \\
& \quad+\frac{1}{2 \pi i} \int_{\operatorname{Re}(s)=x_{0}} \frac{|t|_{\mathrm{A}}^{s / 2-1 / 2} q^{1-g \zeta_{K}}(s) / \zeta_{K}(s+1)}{w-s} \phi(s) d s .
\end{aligned}
$$


The former integral in (3.5) is $O\left(|t|_{\mathbf{A}}^{N}\right) \forall N>0$. We simply push the line of integration to $\operatorname{Re}(s)=-2 N-1$ to obtain this estimate. As for the latter integral,

$$
\begin{aligned}
\frac{1}{2 \pi i} \int_{\operatorname{Re}(s)=x_{0}<\operatorname{Re}(w)} \frac{|t|_{\mathrm{A}}^{s / 2-1 / 2} q^{1-g \zeta_{K}}(s) / \zeta_{K}(s+1)}{w-s} \phi(s) d s \\
=|t|_{\mathrm{A}}^{w / 2-1 / 2} q^{1-g} \frac{\zeta_{K}(w)}{\zeta_{K}(w+1)} \phi(w) \\
+\frac{1}{2 \pi i} \int_{\operatorname{Re}(s)=x_{1}>\operatorname{Re}(w)} \frac{|t|_{\mathrm{A}}^{s / 2-1 / 2} q^{1-g} \zeta_{K}(s) / \zeta_{K}(s+1)}{w-s} \phi(s) d s .
\end{aligned}
$$

The latter integral is $O\left(|t|_{\mathrm{A}}^{N}\right), \forall N>0$. Hence the dominant term is

$$
|t|_{\mathbf{A}}^{w / 2-1 / 2} q^{1-g} \frac{\zeta_{K}(w)}{\zeta_{K}(w+1)} \phi(w)=O\left(|t|_{\mathbf{A}}^{(\operatorname{Re}(w)-1) / 2}\right) .
$$

(iii) We first introduce a notation that will come in handy throughout the rest of §3. For two complex functions $\phi_{1}(w)$ and $\phi_{2}(w)$, we will write $\phi_{1}(w) \sim \phi_{2}(w)$ if $\phi_{1}(w)-\phi_{2}(w)$ is analytic in a half-plane $\operatorname{Re}(w)>\sigma$ for some $\sigma<1$.

The Fourier expansion for the Eisenstein series $E(g, s)=\sum_{a \in K} C_{a}(t, s) \psi(a u)$ gives rise to the Fourier expansion for the "smoothed" Eisenstein series

$$
\mathscr{E}(g, \phi, w)=\sum_{a \in K} \mathscr{C}_{a}(t, \phi, w) \psi(a u),
$$

where

$$
\mathscr{C}_{a}(t, \phi, w)=\frac{1}{2 \pi i} \int_{\operatorname{Re}(s)=x_{0}<\operatorname{Re}(w)} \frac{C_{a}(t, s)}{w-s} \phi(s) d s .
$$

By Proposition 3.2(ii), all but finitely many $\mathscr{C}_{a}(t, \phi, w)=0$, and if $a \neq 0, \mathscr{C}_{a}(t, \phi, w)$ is an analytic function of $w$ in the half-plane $\operatorname{Re}(w)>-\frac{1}{2}$. Hence $\mathscr{E}(g, \phi, w) \sim$ $\mathscr{C}_{0}(t, \phi, w)$. Now

$$
\begin{aligned}
\mathscr{C}_{0}(t, \phi, w)= & |t|^{(w-1) / 2} q^{1-g} \frac{\zeta_{K}(w)}{\zeta_{K}(w+1)} \phi(w) \\
& +\frac{1}{2 \pi i} \int_{\operatorname{Re}(s)=x_{0}<\operatorname{Re}(w)} \frac{|t|_{\mathrm{A}}^{-s / 2-1 / 2}}{w-s} \phi(s) d s \\
& +\frac{1}{2 \pi i} \int_{\operatorname{Re}(s)=x_{1}>\operatorname{Re}(w)} \frac{|t|_{\mathrm{A}}^{s / 2-1 / 2} q^{1-g} \zeta_{K}(s) / \zeta_{K}(s+1)}{w-s} \phi(s) d s .
\end{aligned}
$$

Both integrals in this expression are entire functions of $w$. Hence $\mathscr{E}(g, \phi, w)$ $|t|_{\mathbf{A}}^{(w-1) / 2} q^{1-\not g} \zeta_{K}(w) \phi(w) / \zeta_{K}(w+1)$ and

$$
\lim _{w \rightarrow 1}\left(1-q^{1-w}\right) \mathscr{E}(g, \phi, w)=q^{1-g} \frac{\operatorname{Res}_{q} \zeta_{K}}{\zeta_{K}(2)} \phi(1)
$$


The limit converges uniformly in $g, g \in \Theta$, since for all but a compact subset of $Z_{\mathrm{A}} \backslash \theta / B_{K}$, every $\mathscr{C}_{a}(t, \phi, w)$ other than $\mathscr{C}_{0}(t, \phi, w)$ is zero.

(iv) Since $\Theta$ contains a fundamental domain of $G_{K}$ in $G_{\mathrm{A}}$ and

$$
\lim _{w \rightarrow 1}\left(1-q^{1-w}\right) \mathscr{E}(g, \phi, w)
$$

converges uniformly in $\Theta$,

$$
\begin{aligned}
\lim _{w \rightarrow 1}\left(1-q^{1-w}\right) & \int_{G_{\mathrm{A}} / G_{K}} f(g) \mathscr{E}(g, \phi, w) d g \\
& =\int_{G_{\mathrm{A}} / G_{K}} \lim _{w \rightarrow 1}\left(\left(1-q^{1-w}\right) \mathscr{E}(g, \phi, w)\right) f(g) d g \\
& =q^{1-g} \frac{\operatorname{Res}_{q} \zeta_{K}}{\zeta_{K}(2)} \phi(1) \int_{G_{\mathrm{A}} / G_{K}} f(g) d g . \quad \text { Q.E.D. }
\end{aligned}
$$

For $f \in \mathscr{S}\left(V_{\mathbf{A}}\right)$, set

$$
I_{i}^{n}(\omega, f ; \phi, w)=\int_{G_{\mathrm{A}}^{n} / G_{K}} \omega(\operatorname{det}(g)) \sum_{x \in S_{K, i}} f(g \circ x) \mathscr{E}(g, \phi, w) d g, \quad i=0,1,2,
$$

and

$$
\begin{aligned}
& I^{n}(\omega, f ; \phi, w) \\
& \quad=\int_{G_{A}^{n} / G_{K}} \omega(\operatorname{det}(g))\left(\omega_{-2}(\operatorname{det}(g)) \sum_{x \in S_{K}} \hat{f}\left(g^{\prime} \circ x\right)-\sum_{x \in S_{K}} f(g \circ x)\right) \mathscr{E}(g, \phi, w) d g .
\end{aligned}
$$

Then Lemmas 3.1 and 3.2(ii), (iv), imply

Proposition 3.3. The integrals $I_{i}^{n}(\omega, f ; \phi, w)$ converge absolutely and locally uniformly in $w$ in the half-plane $\operatorname{Re}(w)>3$, the integral $I^{n}(\omega, f ; \phi, w)$ converges absolutely and locally uniformly in $w$ in the half-plane $\operatorname{Re}(w)>1$,

$$
\left.I^{n}(\omega, f ; \phi, w)=\sum_{i=0}^{2} I_{i}^{-n}\left(\omega_{2} \omega^{-1}, \hat{f} ; \phi, w\right)-I_{i}^{n}(\omega, f ; \phi, w)\right), \quad \operatorname{Re}(w)>3,
$$

and

$$
I^{n}(\omega, f)=\left(q^{1-g} \phi(1) \frac{\operatorname{Res} \zeta_{K}}{\zeta_{K}(2)}\right)^{-1} \lim _{w \rightarrow 1}\left(1-q^{1-w}\right) I^{n}(\omega, f ; \phi, w) .
$$

Thus we are able to break up the integral $I^{n}(\omega, f)$ into a sum of simpler integrals.

3.5 Evaluation of the integrals $I_{i}^{n}(\omega, f ; \phi, w)$ gives rise to certain invariant distributions on $\mathscr{S}\left(V_{\mathrm{A}}\right)$. Before undertaking the actual calculation, we would like to describe these distributions and briefly describe some of their properties.

The distribution $\Sigma_{0}$ that arises out of $I_{0}^{n}$ is rather simple: $\Sigma_{0}(f)=f(0)$. The description of distributions, arising out of $I_{1}^{n}$ and $I_{2}^{n}$, is more complicated. We begin with an averaging operator $M_{\omega}$, defined by

$$
\left(M_{\omega} f\right)(x)=\int_{k} \omega(\operatorname{det}(k)) f(k \circ x) d k,
$$

where $\omega \in \Omega$ and $f \in \mathscr{S}\left(V_{\mathbf{A}}\right)$. 
The averaging operator has a number of pleasant properties. Among them are

(i) for any $f \in \mathscr{S}\left(V_{\mathbf{A}}\right), M_{\omega} f \in \mathscr{S}\left(V_{\mathbf{A}}\right)$;

(ii) $M_{\omega} f=M_{\tilde{\omega}} f, \omega=\tilde{\omega} \omega_{s}$;

(iii) $M_{\omega}\left(M_{\omega} f\right)=M_{\omega} f$;

(iv) $M_{\omega} \hat{f}=\overline{M_{\bar{\omega}} f}$.

The averaging operator we will encounter most often is $M_{1}$ :

$$
\left(M_{1} f\right)(x)=\int_{k} f(k \circ x) d k
$$

Next we define two operators $T_{1}$ and $T_{2}: \mathscr{S}\left(V_{\mathbf{A}}\right) \rightarrow \mathscr{S}(\mathbf{A})$, given as follows: for $t \in \mathbf{A}$,

$$
\begin{aligned}
& \left(T_{1} f\right)(t)=f(0,0,0, t) \\
& \left(T_{2} f\right)(t)=\int_{\mathbf{A}} f(0,0, t, u) d u
\end{aligned}
$$

Finally, we recall the definition of the classical zeta function $\zeta(\cdot, \Phi): \Omega \rightarrow \mathrm{C}$, first studied by Tate in his thesis [28]:

$$
\zeta(\omega, \Phi)=\int_{\mathbf{A}^{\times}} \omega(t) \Phi(t) d^{\times} t
$$

Here $\Phi$ is a Schwartz-Bruhat function on $\mathbf{A}$.

The singular distributions that arise in the integrals $I_{1}^{n}$ and $I_{2}^{n}$ are, respectively,

$$
\Sigma_{1}(\omega, f)=\zeta\left(\omega, T_{1}\left(M_{\bar{\omega}} f\right)\right)=\int_{\mathbf{A}^{\times}} \omega(t) M_{\bar{\omega}} f(0,0,0, t) d^{\times} t
$$

and

$$
\Sigma_{2}(\omega, f)=\zeta\left(\omega, T_{2}\left(M_{\bar{\omega}} f\right)\right)=\int_{\mathbf{A}^{\times}} \int_{\mathbf{A}} \omega(t) M_{\bar{\omega}} f(0,0, t, u) d u d^{\times} t
$$

In the evaluations that follow, we will use slightly more suggestive notations: $\Sigma_{2}(s, f)$ for $\Sigma_{2}\left(\omega_{s}, f\right)$ and $\Sigma_{1}(\tilde{\omega}, s, f)$ for $\Sigma_{1}\left(\tilde{\omega} \omega_{s}, f\right)$.

The analytic properties of $\zeta(\omega, \Phi)$ are well known. In particular, the integral that defines $\zeta(\omega, \Phi)$ converges absolutely and locally uniformly in $\omega$ when $\operatorname{Re}(\omega)>1$ and can be analytically continued in a rational function on the entire space $\Omega$. More precisely,

$$
\zeta(\omega, \Phi)=P(\omega, \Phi)+\delta(\omega)\left(\frac{\hat{\Phi}(0)}{1-q^{1-s}}-\frac{\Phi(0)}{1-q^{-s}}\right),
$$

where $P(\omega, \Phi)$ is a polynomial in $\left(q^{s}, q^{-s}\right), \omega=\tilde{\omega} \omega_{s}$, and $\delta(\omega)=1$ if $\tilde{\omega}=1$ and $\delta(\omega)=0$ if $\tilde{\omega} \neq 1$. Proofs of these facts can be found in [33] or in the original work of Tate [28].

An immediate consequence of these results of Tate is the following proposition. 
Proposition 3.4. The integrals, defining $\Sigma_{1}(\omega, f)$ and $\Sigma_{2}(\omega, f)$, converge absolutely and locally uniformly in $\omega, \operatorname{Re}(\omega)>1$, and can be analytically continued to rational functions on the entire space $\Omega$. More precisely,

$$
\begin{aligned}
& \Sigma_{1}(\omega, f)=P_{1}(\omega, f)+\delta(\omega)\left(\frac{\left.\left(\widehat{T_{1}\left(M_{\bar{\omega}} f\right.}\right)\right)(0)}{1-q^{1-s}}-\frac{\left(T_{1}\left(M_{\bar{\omega}} f\right)\right)(0)}{1-q^{-s}}\right), \\
& \Sigma_{2}(\omega, f)=P_{2}(\omega, f)+\delta(\omega)\left(\frac{\left.\left(\widehat{T_{2}\left(M_{\bar{\omega}} f\right.}\right)\right)(0)}{1-q^{1-s}}-\frac{\left(T_{2}\left(M_{\bar{\omega}} f\right)\right)(0)}{1-q^{-s}}\right),
\end{aligned}
$$

where $P_{1}$ and $P_{2}$ are polynomials in $\left(q^{s}, q^{-s}\right), \omega=\tilde{\omega} \omega_{s}$.

The distributions that appear in $I_{1}^{n}$ are slight variations on $\Sigma_{1}$. Let $\mathbf{A}^{(n, 3)}=\{t \in$ $\left.\left.\mathbf{A}^{\times}|| t\right|_{\mathbf{A}}=q^{3 m+n}, m \in \mathbf{Z}\right\}$ and set

$$
\Sigma_{1}^{(n, 3)}(\omega, f)=\int_{\mathbf{A}^{(n, 3)}} \omega(t)\left(M_{\bar{\omega}} f\right)(0,0,0, t) d^{\times} t .
$$

This is precisely the distribution that appears in $I_{1}^{n}$.

It is a rather trivial matter to extract $\Sigma_{1}^{(i, 3)}$ out of $\Sigma_{1}$. Thus Proposition 3.3 implies that

$$
\Sigma_{1}^{(i, 3)}(\omega, f)=P_{1}^{(i)}(\omega, f)+\delta(\omega)\left(\frac{\left.\left(\widehat{T_{1}\left(M_{\bar{\omega}} f\right.}\right)\right)(0)}{1-q^{3-3 s}} q^{i(s-1)}-\frac{\left(T_{1}\left(M_{\bar{\omega}} f\right)\right)(0)}{1-q^{-3 s}} q^{i s}\right)
$$

$i=0,1,2$, where the $P_{1}^{(i)}$ 's are polynomials in $\left(q^{s}, q^{-s}\right)$.

Distributions $\Sigma_{1}(\omega, f)$ and $\Sigma_{2}(\omega, f)$ are not, in general, invariant. However, the distributions $\Sigma_{0}(f)=f(0), \quad \Sigma_{1}\left(\tilde{\omega}, \frac{2}{3}, f\right)$ where $\tilde{\omega}^{3}=1$, and $\Sigma_{2}(-1, f)$ are. Their invariance follows from the fact that they are essentially residues of the adelic zeta function $Z(\omega, f)$, which obviously is a $G_{\mathrm{A}^{-}}$-invariant. Unfortunately, we do not know if these three distributions exhaust the list of singular invariant distributions on $V_{\mathbf{A}}$. We challenge the reader to find an answer to this question.

For a more detailed discussion of singular invariant distributions on $V_{\mathrm{A}}$, we refer the reader to [36].

3.6 We are now ready to evaluate the integrals $I_{i}^{n}(\omega, f ; \phi, w), i=0,1,2$. Of course, we are not interested in these integrals per se; all we want is

$$
\lim _{w \rightarrow 1}\left(1-q^{1-w}\right) I^{n}(\omega, f ; \phi, w) .
$$

Hence our use of the equivalence relation " ", introduced in the proof of Lemma 3.2(iii). We first prove

Proposition 3.5.

$$
I_{0}^{n}(\omega, f ; \phi, w) \sim \delta(\omega) \omega\left(\pi^{n}\right) f(0) \frac{q^{n(1-w) / 2}}{1-q^{1-n^{\prime}}} \phi(w),
$$

where $\pi$ is the uniformizer of $\mathbf{A}^{\times}$of $\$ 2.2$. 


\section{Proof.}

$$
\begin{aligned}
I_{0}^{n}(\omega, f ; \phi, w) & =\int_{G_{\mathbf{A}}^{n} / G_{K}} \omega(\operatorname{det}(g)) f(0) \mathscr{E}(g, \phi, w) d g \\
& =\int_{G_{\mathbf{A}}^{n} / G_{K}} \omega(\operatorname{det}(g)) f(0)\left(\frac{1}{2 \pi i} \int_{\operatorname{Re}(s)=x} \frac{\sum_{\gamma \in G_{K} / B_{K}}|t(g \gamma)|_{\mathbf{A}}^{s / 2-1 / 2}}{w-s} \phi(g) d s\right) d g \\
& =\int_{G_{\mathbf{A}}^{n} / B_{K}} \omega(\operatorname{det}(g)) f(0)\left(\frac{1}{2 \pi i} \int_{\operatorname{Re}(s)=x} \frac{|t(g)|_{\mathbf{A}}^{-s / 2-1 / 2}}{w-s} \phi(s) d s\right) d g \\
& =f(0) \int_{k} \omega(\operatorname{det}(k)) d k \int_{\mathbf{A}^{\times} / K^{\times}} \int_{\mathbf{A}^{\times} / K^{\times}} \int_{\mathbf{A} / K} \omega\left(t_{1}^{2} t\right) \\
& \times\left(\frac{1}{2 \pi i} \int_{\operatorname{Re}(s)=x_{0}} \frac{|t|_{\mathbf{A}}^{-s / 2-1 / 2}}{w-s} \phi(s) d s\right)|t|_{\mathbf{A}} d u d^{\times} t d^{\times} t_{1} \\
= & \varepsilon(\omega) f(0) \int_{\mathbf{A}^{\times} / K^{\times}} \int_{\mathbf{A}^{\times} / K^{\times}} \omega\left(t_{1}^{2} t\right)\left(\frac{1}{2 \pi i} \int_{\operatorname{Re}(s)=x_{0}} \frac{|t|_{\mathbf{A}}^{-(s+1) / 2}}{w-s} \phi(s) d s\right) d^{\times} t d^{\times} t_{1},
\end{aligned}
$$

where

$$
\varepsilon(\omega)= \begin{cases}1 & \text { if } \omega=1 \text { on } \mathbf{A}(\varnothing) \\ 0 & \text { otherwise. }\end{cases}
$$

Replace $t$ by $t_{1}^{2} t$ and note that $|t|_{\mathbf{A}}=q^{n}\left|t_{1}\right|_{\mathbf{A}}^{-2}$. Then (3.6) becomes

$$
\varepsilon(\omega) f(0) \int_{\mathbf{A}^{n} / K^{\times}} \omega(t) d^{\times} t \int_{\mathbf{A}^{\times} / K^{\times}}\left(\frac{1}{2 \pi i} \int_{\operatorname{Re}(s)=x_{0}} \frac{q^{-n(s-1) / 2}\left|t_{1}\right|_{\mathbf{A}}^{s-1}}{w-s} \phi(s) d s\right) d^{\times} t_{1} .
$$

Here $\mathbf{A}^{n}=\left\{\left.t \in \mathbf{A}^{\times}|| t\right|_{\mathbf{A}}=q^{n}\right\}$.

Now, $\int_{\mathbf{A}^{n} / K^{\times}} \omega(t) d^{\times} t=\delta(\omega) \omega\left(\pi^{n}\right)$ and $\delta(\omega) \varepsilon(\omega)=\delta(\omega)$. Hence (3.7) equals

$$
\begin{aligned}
& \delta(\omega) \omega\left(\pi^{n}\right) f(0) \int_{\mathbf{A}^{\times} / K^{\times}}\left(\frac{1}{2 \pi i} \int_{\operatorname{Re}(s)=x_{0}} \frac{q^{-n(s-1) / 2}|t|_{\mathbf{A}}^{s-1}}{w-s} \phi(s) d s\right) d^{\times} t \\
&= \delta(\omega) \omega\left(\pi^{n}\right) f(0)\left(\int_{\mathbf{A}^{+} / K^{\times}}\left(\frac{1}{2 \pi i} \int_{\operatorname{Re}(s)=x_{0}} \frac{q^{-n(s-1) / 2}|t|_{\mathbf{A}}^{s-1}}{w-s} \phi(s) d s\right) d^{\times} t\right) \\
&+\int_{\mathbf{A}^{-} / K^{\times}}\left(\frac{1}{2 \pi i} \int_{\operatorname{Re}(s)=x_{0}} \frac{q^{-n(s-1) / 2}|t|_{\mathbf{A}}^{s-1}}{w-s} \phi(s) d s\right) d^{\times} t
\end{aligned}
$$

where $\mathbf{A}^{+}=\left\{\left.t \in \mathbf{A}^{\times}|| t\right|_{\mathbf{A}}>1\right\}$ and $\mathbf{A}^{-}=\left\{\left.t \in \mathbf{A}^{\times}|| t\right|_{\mathbf{A}} \leqslant 1\right\}$. 
The first of the two integrals in (3.8)

$$
\begin{aligned}
\int_{\mathbf{A}^{+} / K^{\times}}\left(\frac{1}{2 \pi i} \int_{\operatorname{Re}(s)=x_{0}} \frac{q^{-n(s-1) / 2}|t|_{\mathbf{A}}^{s-1}}{w}-s(s) d s\right) d^{\times} t \\
\quad=\sum_{m=1}^{\infty} \frac{1}{2 \pi i} \int_{\operatorname{Re}(s)=x_{0}} \frac{q^{(m-n / 2)(s-1)}}{w-s} \phi(s) d s .
\end{aligned}
$$

Each of the integrals in the latter sum is an entire function of $w$; moreover,

$$
\begin{aligned}
\frac{1}{2 \pi i} \int_{1<\operatorname{Re}(s)=x_{0}<\operatorname{Re}(w)} & \frac{q^{(m-n / 2)(s-1)}}{w-s} \phi(s) d s \\
= & \frac{1}{2 \pi i} \int_{\operatorname{Re}(s)=-\alpha+1} \frac{q^{(m-n / 2)(s-1)}}{w-s} \phi(s) d s=O\left(q^{-(m-n / 2) \alpha}\right)
\end{aligned}
$$

for any $\alpha>0$. Hence the sum in (3.9) converges uniformly and is therefore an entire function of $w$. Hence

$$
\begin{aligned}
& I_{0}^{n}(\omega, f ; \phi, w) \sim \delta(\omega) \omega\left(\pi^{n}\right) f(0) \int_{\mathbf{A}^{-} / K^{\times}} \frac{1}{2 \pi i} \int_{\operatorname{Re}(s)=x_{0}} \frac{q^{-n(s-1) / 2}|t|_{\mathrm{A}}^{s-1}}{w-s} \phi(s) d s d^{\times} t \\
& =\delta(\omega) \omega\left(\pi^{n}\right) f(0) \sum_{m=0}^{\infty} \frac{1}{2 \pi i} \int_{1<\operatorname{Re}(s)=x_{0}<\operatorname{Re}(w)} \frac{q^{-n(s-1) / 2} q^{-m(s-1)}}{w-s} \phi(s) d s \\
& =\delta(\omega) \omega\left(\pi^{n}\right) f(0) \frac{1}{2 \pi i} \int_{1<\operatorname{Re}(s)=x_{0}<\operatorname{Re}(w)} \frac{q^{-n(s-1) / 2}\left(1-q^{1-s}\right)^{-1}}{w-s} \phi(s) d s \\
& =\delta(\omega) \omega\left(\pi^{n}\right) f(0)\left(\frac{q^{n(1-w) / 2}}{1-q^{1-w}} \phi(w)\right. \\
& \left.\quad+\frac{1}{2 \pi i} \int_{\operatorname{Re}(s)=x_{1}>\operatorname{Re}(w)} \frac{q^{-n(s-1) / 2}\left(1-q^{1-s}\right)^{-1}}{w-s} \phi(s)\right) d s \\
& \sim \delta(\omega) \omega\left(\pi^{n}\right) f(0) \frac{q^{n(1-w) / 2}}{1-q^{1-w}} \phi(w)
\end{aligned}
$$

since the integral over $\operatorname{Re}(s)=x_{1}>\operatorname{Re}(w)$ is an entire function of $w$. Q.E.D.

As a corollary to Proposition 3.5, we note that the volume of a fundamental

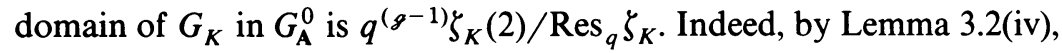

$$
q^{1-g_{\phi}}(1) \frac{\operatorname{Res}_{q} \zeta_{K}}{\zeta_{K}(2)} \int_{G_{\mathrm{A}}^{0} / G_{K}} d g=\lim _{w \rightarrow 1}\left(1-q^{1-w}\right) \int_{G_{\mathrm{A}}^{0} / G_{K}} \mathscr{E}(g, \phi, w) d g=\phi(1)
$$

The latter identity is a particular case of Proposition 3.5 with $f(0)=1$ and $\omega=1$. Hence the corollary.

We next turn to the integral $I_{1}^{n}(\omega, f ; \phi, w)$.

Proposition 3.6.

$$
I_{1}^{n}(\omega, f ; \phi, w)
$$

$$
\sim \delta\left(\omega^{3}\right) \omega\left(\pi^{n}\right) q^{-n(w+1) / 6} q^{1-g} \frac{\zeta_{K}(w)}{\zeta_{K}(w+1)} \phi(w) \Sigma_{1}^{(-n, 3)}\left(f, \tilde{\omega}^{-1}, \frac{w+1}{3}\right) .
$$


Proof. Since $S_{K, 1}=\bigcup_{\gamma \in G_{K} / B_{K}} \bigcup_{a \in K^{\times}} \gamma \circ(0,0,0, a)$,

$$
\begin{aligned}
I_{1}^{n}(\omega, f ; \phi, w)= & \int_{G_{\mathbf{A}}^{n} / G_{K}} \omega(\operatorname{det}(g)) \sum_{x \in S_{K, 1}} f(g \circ x) \mathscr{E}(g, \phi, w) d g \\
= & \int_{G_{\mathbf{A}}^{n} / B_{K}} \omega(\operatorname{det}(g)) \sum_{a \in K^{\times}} f(g \circ(0,0,0, a)) \mathscr{E}(g, \phi, w) d g \\
= & \int_{k} \int_{\mathbf{A}^{\times} / K^{\times}} \int_{\mathbf{A}^{\times} / K^{\times}} \int_{\mathbf{A} / K} \omega(\operatorname{det}(k)) \omega\left(t_{1}^{2} t\right) \\
& \cdot \sum_{a \in K^{\times}} f\left(k d\left(t_{1}^{2}\right) a(t) n(u) \circ(0,0,0, a)\right) \\
& \cdot \mathscr{E}(a(t) n(u), \phi, w)|t|_{\mathbf{A}} d u d^{\times} t d^{\times} t_{1} d k .
\end{aligned}
$$

We now note that

(i) integration with respect to $d k$ replaces $f$ by $M_{\omega} f$;

(ii) $d\left(t_{1}\right) a(t) n(u) \circ(0,0,0, a)=\left(0,0,0, t_{1} t^{2} a\right)$ is independent of $u$. Hence integration with respect to $d u$ replaces $\mathscr{E}(a(t) n(u), \phi, w)$ by $\mathscr{C}_{0}(t, \phi, w)$;

(iii)

$$
\int_{\mathbf{A}^{\times} / K^{\times}} \sum_{a \in K^{\times}} f\left(0,0,0, t_{1} t^{2} a\right) d^{\times} t_{1}=\int_{\mathbf{A}^{\times}} f\left(0,0,0, t_{1} t^{2}\right) d^{\times} t_{1} .
$$

Hence

$$
\begin{aligned}
I_{1}^{n}(\omega, f ; \phi, w)= & \int_{\mathbf{A}^{\times} / K^{\times}} \int_{\mathbf{A}^{\times}} \omega\left(t_{1}^{2} t\right)\left(M_{\omega} f\right)\left(0,0,0, t_{1}^{2} t\right) \mathscr{C}_{0}(t, \phi, w)|t|_{\mathbf{A}} d^{\times} t_{1} d^{\times} t \\
= & \int_{\substack{\mathbf{A}^{\times} / K^{\times} \\
\left|t_{1}^{2}\right|_{\mathbf{A}}=q^{\prime \prime}}} \int_{\mathbf{A}^{\times}} \omega\left(t_{1}^{2} t\right)\left(M_{\omega} f\right)\left(0,0,0, t_{1} t^{2}\right) \\
& \cdot\left(\frac{1}{2 \pi i} \int \frac{|t|_{\mathbf{A}}^{(-s+1) / 2}+q^{1-\gamma}|t|_{\mathbf{A}}^{(s+1) / 2} \zeta_{K}(s) / \zeta_{K}(s+1)}{w-s} \phi(s) d s\right) d^{\times} t_{1} d^{\times} t .
\end{aligned}
$$

Since $t_{1}^{2} t=\left(t_{1} t^{2}\right)^{2} t^{-3}$ and $|t|_{\mathbf{A}}^{3}=q^{-n}\left|t_{1}^{2} t\right|_{\mathbf{A}}^{2}$, the change of variables $t_{1} \rightarrow t_{1} t^{2}$ transforms (3.10) into

$$
\begin{aligned}
& \int_{\mathbf{A}^{\times}, k^{\times \times}} \int_{\mathbf{A}^{\times}} \omega\left(t_{1}^{2} t^{-e}\right)\left(M_{\omega} f\right)\left(0,0,0, t_{1}\right) \\
& |r|_{A}^{3}=q^{-n}\left|r_{1}\right|_{A}^{2} \\
& \cdot\left(\frac{1}{2 \pi i} \int_{\left.\mathrm{R}((-))=x_{0}\right)} \frac{q^{n(s-1) / 6}\left|t_{1}\right|_{\mathrm{A}}^{(-s+1) / 3}}{w-s} \phi(s) d s\right. \\
& \left.+\frac{1}{2 \pi i} \int_{\operatorname{Re}(s)=x_{6}} \frac{q^{1-g} q^{-n(s+1) / 6}\left|z_{1}\right|_{A}^{(s+1) / 3} \zeta_{K}(s) / \zeta_{K}(s+1)}{w-s} \phi(s) d s\right) d^{\times} t_{1} d^{\times} t .
\end{aligned}
$$


We note that in the above integral $t_{1}$ ranges over $\mathbf{A}^{(-n, 3)}=\left\{\left.t \in \mathbf{A}|| t\right|_{\mathbf{A}}=q^{3 m-n}\right.$, $m \in \mathbf{Z}\}$ and $\omega\left(t_{1}^{2} t^{-3}\right)=\omega\left(\pi^{n}\right) \tilde{\omega}\left(t_{1}^{2} t^{-3}\right)$. Changing the order of integration and integrating with respect to $d^{\times} t$, we obtain

$$
\begin{aligned}
I_{1}^{n}(\omega, f ; \phi, w) & \delta\left(\omega^{3}\right) \omega\left(\pi^{n}\right) \int_{\mathbf{A}^{(-n, 3)}} \tilde{\omega}\left(t^{2}\right)\left(M_{\omega} f\right)(0,0,0, t) \\
& \cdot\left(\frac{1}{2 \pi i} \int_{\operatorname{Re}(s)=x_{0}} \frac{q^{n(s-1) / 6}|t|_{\mathrm{A}}^{(-s+1) / 3}}{w-s} \phi(s) d s\right) d^{\times} t \\
+ & \delta\left(\omega^{3}\right) \omega\left(\pi^{n}\right) \int_{\mathbf{A}^{(-n, 3)}} \tilde{\omega}\left(t^{2}\right)\left(M_{\omega} f\right)(0,0,0, t) \\
& \cdot\left(\frac{1}{2 \pi i} \int_{\operatorname{Re}(s)=x_{0}} \frac{q^{1-g} q^{-n(s+1) / 6}|t|_{\mathrm{A}}^{(s+1) / 3} \zeta_{K}(s) / \zeta_{K}(s+1)}{w-s} \phi(s) d s\right) d^{\times} t .
\end{aligned}
$$

The former integral in (3.12) equals

$$
\sum_{m=-\infty}^{\infty} \int_{\mathbf{A}^{3 m-n}} \tilde{\omega}^{2}(t)\left(M_{\omega} f\right)(0,0,0, t) d^{\times} t \frac{1}{2 \pi i} \int_{\operatorname{Re}(s)=x_{0}} \frac{q^{(n / 6-m)(s-1)}}{w-s} \phi(s) d s .
$$

Now, $M_{\omega} f$ is a locally constant function with a compact support. Therefore

$$
\int_{\mathbf{A}^{3 m-n}} \tilde{\omega}\left(t^{2}\right)\left(M_{\omega} f\right)(0,0,0, t) d^{\times} t= \begin{cases}0 & \text { when } m \text { is large positive, } \\ O\left(q^{-3 m}\right) & \text { when } m \text { is large negative. }\end{cases}
$$

The integral $\int_{\operatorname{Re}(s)=x}\left(q^{(n / 6-m)(s-1)} /(w-s)\right) \phi(s) d s$ is an entire function of $w$; moreover, when $m$ is large and negative this integral is $O\left(q^{m \alpha}\right)$ for any $\alpha>0$. This estimate is obtained by pushing the line of integration to $\operatorname{Re}(s)=-\alpha+1$. Hence the sum in (3.13) converges uniformly and therefore represents an entire function of $w$. Thus

$$
\begin{aligned}
& I_{1}^{n}(\omega, f ; \phi, w) \sim \delta\left(\omega^{3}\right) \omega\left(\pi^{n}\right) \int_{A^{(-n, 3)}} \tilde{\omega}\left(t^{2}\right)\left(M_{\omega} f\right)(0,0,0, t) \\
& \quad \cdot\left(\frac{1}{2 \pi i} \int_{\operatorname{Re}(s)=x_{0}<\operatorname{Re}(w)} \frac{q^{1-8} q^{-n(s+1) / 6}|t|_{A}^{(s+1) / 3} \zeta_{K}(s) / \zeta_{K}(s+1)}{w-s} \phi(s) d s\right) d^{\times} t \\
& =\delta\left(\omega^{3}\right) \omega\left(\pi^{n}\right) \int_{\mathbf{A}^{(-n .3)}} \tilde{\omega}\left(t^{2}\right)\left(M_{\omega} f\right)(0,0,0, t) \\
& \quad \cdot\left(q^{1-8} q^{-n(w+1) / 6} \frac{\zeta_{K}(w)}{\zeta_{K}(w+1)} \phi(w)|t|_{A}^{(w+1) / 3}\right. \\
& \left.\quad+\frac{1}{2 \pi i} \int_{\operatorname{Re}(s)=x_{1}>\operatorname{Re}(w)} \frac{q^{1-g} q^{-n(s+1) / 6}|t|_{A}^{(s+1) / 3} \zeta_{K}(s) / \zeta_{K}(s+1)}{w-s} \phi(s) d s\right) d^{\times} t .
\end{aligned}
$$


The integral over the line $\operatorname{Re}(s)=x_{1}>\operatorname{Re}(w)$ is an entire function of $w$; moreover, when $|t|_{\mathbf{A}}$ is small, it is $O\left(|t|_{\mathbf{A}}^{\alpha}\right)$ for any $\alpha>0$. Hence subsequent integration with respect to $d^{\times} t$ would yield an entire function of $w$, and therefore

$$
\begin{aligned}
I_{1}^{n}(\omega, f ; \phi, w) & \sim \delta\left(\omega^{3}\right) \omega\left(\pi^{n}\right) q^{-n(w+1) / 6} q^{1-g} \frac{\zeta_{K}(w)}{\zeta_{K}(w+1)} \phi(w) \\
& \cdot \int_{\mathbf{A}^{(-n .3)}} \tilde{\omega}\left(t^{2}\right)\left(M_{\omega} f\right)(0,0,0, t)|t|_{\mathbf{A}}^{(w+1) / 3} d^{\times} t \\
= & \delta\left(\omega^{3}\right) \omega\left(\pi^{n}\right) q^{-n(w+1) / 6} q^{1-g} \frac{\zeta_{K}(w)}{\zeta_{K}(w+1)} \phi(w) \Sigma_{1}^{(-n, 3)}\left(f, \tilde{\omega}^{-1}, \frac{w+1}{3}\right) .
\end{aligned}
$$

Q.E.D.

It remains to evaluate the integral $I_{2}^{n}(\omega, f ; \phi, w)$.

Proposition 3.7.

$$
I_{2}^{n}(\omega, f ; \phi, w) \sim \delta(\omega) \omega\left(\pi^{n}\right) q^{n(1-w) / 2} q^{1-g} \frac{\zeta_{K}(w)}{\zeta_{K}(w+1)} \phi(w) \Sigma_{2}(f, w-2) .
$$

Proof. Since $S_{2, K}=\bigcup_{\gamma \in G_{K} / D_{K}} \bigcup_{a \in K^{\times}} \gamma \circ(0,0, a, 0)$,

$$
\begin{aligned}
I_{2}^{n}(\omega, f ; \phi, w) & =\int_{G_{\mathbf{A}}^{n} / G_{K}} \omega(\operatorname{det}(g)) \sum_{x \in S_{2, \kappa}} f(g \circ x) \mathscr{E}(g, \phi, w) d g \\
& =\int_{G_{\mathbf{A}}^{n} / D_{K}} \omega(\operatorname{det}(g)) \sum_{a \in K^{\times}} f(g \circ(0,0, a, 0)) \mathscr{E}(g, \phi, w) d g .
\end{aligned}
$$

Write $g=k d\left(t_{1}\right) a(t) n(u)$ and use the explicit description of the measure $d g$ on $G_{\mathbf{A}} / D_{K}$. Integration with respect to $d k$ replaces $f$ by $M_{\omega} f$,

$$
d\left(t_{1}\right) a(t) n(u) \circ(0,0, a, 0)=\left(0,0, a t_{1} t, a t_{1} t^{2} u\right),
$$

and we therefore obtain

$$
\begin{aligned}
& I_{2}^{n}(\omega, f ; \phi, w)=\int_{\mathbf{A}^{\times} / K^{\times}} \int_{\mathbf{A}^{\times} / K^{\times}} \int_{\mathbf{A}} \omega\left(t_{1}^{2} t\right) \sum_{a \in K^{\times}}\left(M_{\omega} f\right)\left(0,0, a t_{1} t, a t_{1} t^{2} u\right) . \\
& \mid t_{1}^{2} t_{\mathbf{A}}=q^{n} \\
& \cdot \mathscr{E}(a(t) n(u), \phi, w)|t|_{\mathbf{A}} d u d^{\times} t_{1} d^{\times} t \\
& =\int_{\mathbf{A}^{\times} / K^{\times}} \int_{\mathbf{A}^{\times}} \int_{\mathbf{A}} \omega\left(t_{1}^{2} t\right)\left(M_{\omega} f\right)\left(0,0, t_{1} t, t_{1} t^{2} u\right) \\
& \left|t_{1}^{2} t\right|_{\mathbf{A}}=q^{n} \\
& \cdot \mathscr{E}(a(t) n(u), \phi, w)|t|_{\mathbf{A}} d u d^{\times} t_{1} d^{\times} t \\
& =\int_{\mathbf{A}^{\times} / K^{\times}} \int_{\mathbf{A}^{\times}} \int_{\mathbf{A}} \omega\left(t_{1}^{2} t^{-1}\right)\left(M_{\omega} f\right)\left(0,0, t_{1}, u\right) \\
& \left|t_{1}^{2} t^{-1}\right|_{\mathbf{A}}=q^{n} \\
& \cdot \mathscr{E}\left(a(t) n\left(\frac{u}{t_{1} t}\right), \phi, w\right)\left|t_{1}\right|_{\mathbf{A}}^{-1} d u d^{\times} t_{1} d^{\times} t,
\end{aligned}
$$

the last integral being obtained by the change of variables $t_{1} \rightarrow t_{1} t$ and $u \rightarrow t_{1} t^{2} u$. 
Write

where

$$
\mathscr{E}(a(t) n(u), \phi, w)=\sum_{a \in K} \mathscr{C}_{a}(t, \phi, w) \psi(a u)
$$

$$
\mathscr{C}_{a}(t, \phi, w)=\frac{1}{2 \pi i} \int_{\operatorname{Re}(s)=x_{0}<\operatorname{Re}(w)} \frac{C_{a}(t, s)}{w-s} \phi(s) d s
$$

as in the proof of Lemma 3.2(iii), and consider first

$$
\begin{aligned}
& \sum_{a \in K^{\times}} \int_{\mathbf{A}^{\times} / K^{\times}} \int_{\mathbf{A}^{\times}} \int_{\mathbf{A}} \omega\left(t_{1}^{2} t^{-1}\right)\left(M_{\omega} f\right)\left(0,0, t_{1}, u\right) \mathscr{C}_{a}(t, \phi, w) \psi\left(\frac{a u}{t_{1} t}\right)\left|t_{1}\right|_{\mathbf{A}}^{-1} d u d^{\times} t_{1} d^{\times} t . \\
& \left|t_{1}^{2} t^{-1}\right|_{\mathbf{A}}=q^{n}
\end{aligned}
$$

Since $\left|t_{1}\right|_{\mathbf{A}}^{2}=q^{n}|t|_{\mathbf{A}}$ and since $M_{\omega} f$ has a compact support, there exists an $N>0$ such that $\left(M_{\omega} f\right)\left(0,0, t_{1}, u\right)=0$ whenever $|t|_{\mathbf{A}}>q^{N}$. But the number of $a$ 's such that $\mathscr{C}_{a}(t, \phi, w) \neq 0$ for some $t$ with $|t|_{\mathrm{A}} \leqslant q^{N}$ is finite. This is due to the following observations.

(i) For any $t \in \mathbf{A}^{\times}$, the number of $a$ 's such that $\mathscr{C}_{a}(t, \phi, w) \neq 0$ is finite. Moreover, two $t$ 's, belonging to the same $K^{\times} \mathbf{A}^{\times}(\varnothing)$-coset in $\mathbf{A}^{\times}$, yield the same Fourier coefficients $\mathscr{C}_{a}(t, \phi, w)$.

(ii) By Proposition 3.2(ii), $\mathscr{C}_{a}(t, \phi, w)=0$ for all $a \in K^{\times}$when $|t|_{\mathrm{A}} \leqslant q^{1-2 \mathscr{g}}$.

(iii) The set $\left\{t \in \mathbf{A}^{\times} /\left.K^{\times} \mathbf{A}^{\times}(\varnothing)\left|q^{2-2 g} \leqslant\right| t\right|_{\mathbf{A}} \leqslant q^{N}\right\}$ is a finite set.

Hence the sume over $a \in K^{\times}$in (3.14) is actually a finite sum.

$\mathscr{C}_{a}(t, s)$ is analytic in the half-plane $\operatorname{Re}(s)>-\frac{1}{2}$. Therefore each of the integrals in (3.14) is an analytic function of $w, \operatorname{Re}(w)>-\frac{1}{2}$, and hence the whole expression (3.14) is analytic in $w, \operatorname{Re}(w)>-\frac{1}{2}$. Hence

$$
\begin{aligned}
& I_{2}^{n}(\omega, f ; \phi, w) \\
& \sim \int_{\substack{\mathbf{A}^{\times} / K^{\times} \\
\left|t_{1}^{2} t^{-1}\right|_{\mathbf{A}}=q^{n}}} \int_{\mathbf{A}^{\times}} \int_{\mathbf{A}} \omega\left(t_{1}^{2} t^{-1}\right)\left(M_{\omega} f\right)\left(0,0, t_{1}, u\right) \mathscr{C}_{0}(t, \phi, w)\left|t_{1}\right|_{\mathbf{A}}^{-1} d u d^{\times} t_{1} d^{\times} t .
\end{aligned}
$$

We change variable $t \rightarrow t_{1}^{2} t^{-1}$ and replace $\mathscr{C}_{0}(t, \phi, w)$ by its integral formula, replacing $|t|_{\mathbf{A}}$ by $q^{-n}\left|t_{1}\right|_{\mathbf{A}}^{2}$. Integration with respect to $d^{\times} t$ now yields $\delta(\omega) \omega\left(\pi^{n}\right)$. and we therefore obtain

$$
\begin{aligned}
& I_{2}^{n}(\omega, f ; \phi, w) \sim \delta(\omega) \omega\left(\pi^{n}\right) \int_{\mathbf{A}^{\times}} \int_{\mathbf{A}}\left(M_{\omega} f\right)(0,0, t, u) \\
& \quad \cdot\left(\frac{1}{2 \pi i} \int_{\operatorname{Re}(s)=x_{0}} \frac{q^{n(s+1) / 2}|t|_{\mathbf{A}}^{-s-2}}{w-s} \phi(s) d s\right. \\
& \left.\quad+\frac{1}{2 \pi i} \int_{\operatorname{Re}(s)=x_{0}} \frac{q^{n(1-s) / 2}|t|_{\mathbf{A}}^{s-2} q^{1-g} \zeta_{K}(s) / \zeta_{K}(s+1)}{w-s} \phi(s) d s\right) d u d^{\times} t \\
& \sim \delta(\omega) \omega\left(\pi^{n}\right) q^{n(1-w) / 2} q^{1-g} \frac{\zeta_{K}(w)}{\zeta_{K}(w+1)} \phi(w) \\
& \cdot \int_{\mathbf{A}^{\times}} \int_{\mathbf{A}}\left(M_{\omega} f\right)(0,0, t, u)|t|_{\mathbf{A}}^{w-2} d u d^{\times} t .
\end{aligned}
$$


The argument that allows us to make the last transition is exactly the same as the one used in the proof of Proposition 3.6. Finally, since $\delta(\omega) \neq 0$ if and only if $\omega=1$ on $\mathbf{A}^{0}$, the integral in (3.15) is precisely $\Sigma_{2}(f, w-2)$. Hence the proposition. Q.E.D.

3.7 We now return to the main problem at hand: an analytic continuation for the zeta function. First, we combine Propositions 3.5, 3.6, and 3.7 with Proposition 3.3 to obtain

Proposition 3.8 .

$$
\begin{aligned}
& I^{n}(\omega, f ; \phi, w) \sim \delta(\omega) \omega\left(\pi^{n}\right)\left(\omega_{-2}\left(\pi^{n}\right) q^{-n(1-w) / 2} \hat{f}(0)-q^{n(1-w) / 2} f(0)\right) \frac{\phi(w)}{1-q^{1-w}} \\
& \quad+\delta\left(\omega^{3}\right) \omega\left(\pi^{n}\right)\left(\omega_{-2}\left(\pi^{n}\right) q^{n(w+1) / 6} \Sigma_{1}^{(n, 3)}\left(\hat{f}, \tilde{\omega}, \frac{w+1}{3}\right)\right. \\
& \left.\quad-q^{-n(w+1) / 6} \Sigma_{1}^{(-n, 3)}\left(f, \tilde{\omega}^{-1}, \frac{w+1}{3}\right)\right) \\
& \quad \cdot q^{1-g} \frac{\zeta_{K}(w)}{\zeta_{K}(w+1)} \phi(w) \\
& +\delta(\omega) \omega\left(\pi^{n}\right)\left(\omega_{-2}\left(\pi^{n}\right) q^{-n(1-w) / 2} \Sigma_{2}(\hat{f}, w-2)-q^{n\left(1-w^{\prime}\right) / 2} \Sigma_{2}(f, w-2)\right) \\
& \quad \cdot q^{1-g} \frac{\zeta_{K}(w)}{\zeta_{K}(w+1)} \phi(w),
\end{aligned}
$$

and

$$
\begin{aligned}
I^{n}(\omega, f) & =\delta(\omega) \omega\left(\pi^{n}\right) q^{g-1} \frac{\zeta_{K}(2)}{\operatorname{Res}_{q} \zeta_{K}}\left(\omega_{-2}\left(\pi^{n}\right) \hat{f}(0)-f(0)\right) \\
& +\delta\left(\omega^{3}\right) \omega\left(\pi^{n}\right)\left(\omega_{-5 / 3}\left(\pi^{n}\right) \Sigma_{1}^{(n, 3)}\left(\hat{f}, \tilde{\omega}, \frac{2}{3}\right)-\omega_{-1 / 3}\left(\pi^{n}\right) \Sigma_{1}^{(-n .3)}\left(f, \tilde{\omega}^{-1}, \frac{2}{3}\right)\right) \\
& +\delta(\omega) \omega\left(\pi^{n}\right)\left(\omega_{-2}\left(\pi^{n}\right) \Sigma_{2}(\hat{f},-1)-\Sigma_{2}(f,-1)\right) .
\end{aligned}
$$

It is of interest to note that $I^{n}(\omega, f ; \phi, w)$ is an analytic function of $w$ in the half-plane $\operatorname{Re}(w)>1$. Indeed, it is a simple exercise in harmonic analysis to show that the pole of $q^{-n(5-w) / 2} \Sigma_{2}(\hat{f}, w-2)$ at $w=3$ cancels the same pole of $q^{n(1-w) / 2} \Sigma_{2}(f, w-2)$, and that the poles of $q^{-n(5-w) / 2} \Sigma_{2}(\hat{f}, w-2)$ and $q^{n(1-w) / 2} \Sigma_{2}(f, w-2)$ at $w=2$ cancel, respectively, the poles of $q^{-n(w+1) / 6} \Sigma_{1}^{(-n, 3)}(f, \tilde{\omega},(w+1) / 3)$ and $q^{n(w-11) / 6} \Sigma_{1}^{(n, 3)}(\hat{f}, \tilde{\omega},(w+1) / 3)$. This is precisely what ought to happen according to Proposition 3.3.

As a corollary to Proposition 3.8 we write down the value for the integral $I^{0}(\omega, f)$. This value will play an important role in the theorems yet to come.

COROllary 3.8.1.

$$
\begin{aligned}
I^{0}(\omega, f)= & \delta(\omega) q^{g-1} \frac{\zeta_{K}(2)}{\operatorname{Res}_{q} \zeta_{K}}(\hat{f}(0)-f(0)) \\
& +\delta\left(\omega^{3}\right)\left(\Sigma_{1}^{(0,3)}\left(\hat{f}, \tilde{\omega}, \frac{2}{3}\right)-\Sigma_{1}^{(0,3)}\left(f, \tilde{\omega}^{-1}, \frac{2}{3}\right)\right) \\
& +\delta(\omega)\left(\Sigma_{2}(\hat{f},-1)-\Sigma_{2}(f,-1)\right) .
\end{aligned}
$$


We now turn to the integral $I(\omega, f)$ of Proposition 3.1. $I(\omega, f)=\frac{1}{2} I^{0}(\omega, f)+$ $\sum_{n=1}^{\infty} I^{-n}(\omega, f)$ and is therefore easily computable. Hence the following theorem.

THEOREM 3.2. For any $f \in \mathscr{S}\left(V_{\mathbf{A}}\right)$, the zeta function $Z(\omega, f)$ is a rational function of $\omega$. More precisely,

$$
Z(\omega, f)=Z_{+}(\omega, f)+Z_{+}\left(\omega_{2} \omega^{-1}, \hat{f}\right)+I(\omega, f),
$$

where $Z_{+}(\omega, f)$ and $Z_{+}\left(\omega_{2} \omega^{-1}, \hat{f}\right)$ are polynomials in $q^{s}$ and $q^{-s}$, respectively, $\omega=\tilde{\omega} \omega_{s}$, and

$$
\begin{aligned}
& I(\omega, f)=\frac{1}{2} I^{0}(\omega, f)+\delta(\omega)\left[q^{g-1} \frac{\zeta_{K}(2)}{\operatorname{Res}_{q} \zeta_{K}} \hat{f}(0)+\Sigma_{2}(\hat{f},-1)\right] \frac{\omega_{2} \omega^{-1}(\pi)}{1-\omega_{2} \omega^{-1}(\pi)} \\
& +\delta\left(\omega^{3}\right)\left[\Sigma_{1}^{(0,3)}\left(\hat{f}, \tilde{\omega}, \frac{2}{3}\right)+\omega_{-5 / 3} \omega(\pi) \Sigma_{1}^{(1,3)}\left(\hat{f}, \tilde{\omega}, \frac{2}{3}\right)\right. \\
& \left.+\omega_{-5 / 3} \omega\left(\pi^{2}\right) \Sigma_{1}^{(2,3)}\left(\hat{f}, \tilde{\omega}, \frac{2}{3}\right)\right] \\
& \frac{\omega_{5} \omega^{-3}(\pi)}{1-\omega_{5} \omega^{-3}(\pi)} \\
& -\delta\left(\omega^{3}\right)\left[\Sigma_{1}^{(0,3)}\left(f, \tilde{\omega}^{-1}, \frac{2}{3}\right)+\omega_{-1 / 3} \omega(\pi) \Sigma_{1}^{(2,3)}\left(f, \tilde{\omega}^{-1}, \frac{2}{3}\right)\right. \\
& \left.+\omega_{-1 / 3} \omega\left(\pi^{2}\right) \Sigma_{1}^{(1,3)}\left(f, \tilde{\omega}^{-1}, \frac{2}{3}\right)\right] \\
& \frac{\omega_{1} \omega^{-3}(\pi)}{1-\omega_{1} \omega^{-3}(\pi)} \\
& -\delta(\omega)\left[q^{g-1} \frac{\zeta_{K}(2)}{\operatorname{Res}_{q} \zeta_{K}} f(0)+\Sigma_{2}(f,-1)\right] \frac{\omega^{-1}(\pi)}{1-\omega^{-1}(\pi)},
\end{aligned}
$$

the value of $I^{0}(\omega, f)$ being given in Corollary 3.8.1.

To rewrite $I(\omega, f)$ in terms of $q^{-s}$, simply observe that $\omega^{-1}(\pi)=q^{-s}$.

A closer look at $I(\omega, f)$ reveals that the poles of $Z(\omega, f)$ are all simple and occur at $\omega=\omega_{2}, \omega=\tilde{\omega} \omega_{5 / 3}, \omega=\tilde{\omega} \omega_{1 / 3}$, and $\omega=1$, where $\tilde{\omega}$ is a character on $\mathbf{A}^{\times} / K^{\times}$ that satisfies $\tilde{\omega}^{3}=1$. While the expressions for the residues at these poles may look forbidding, in reality they are not. Thus in a future paper we will compute the residues of $Z(\omega, f)$ at $\omega=\omega_{2}$ for a certain family of functions $f$, which will in turn lead to a result on the density of discriminants of cubic extensions of a function field. As for now, we would like to list two direct consequences of Theorem 3.2.

Corollary 3.2.1. For any $g \in G_{\mathbf{A}}, f \in \mathscr{S}\left(V_{\mathbf{A}}\right)$, and $\omega \in \Omega$ such that $\delta\left(\omega^{3}\right)=1$,

(i) $\Sigma_{1}^{(i, 3)}\left(g \circ f, \tilde{\omega}, \frac{2}{3}\right)=\tilde{\omega}^{-1}(\operatorname{det}(g))|\operatorname{det}(g)|_{A}^{1 / 3} \Sigma_{1}^{(i, 3)}\left(f ; \tilde{\omega}, \frac{2}{3}\right)$.

(ii) $\Sigma_{2}(g \circ f,-1)=\Sigma_{2}(f,-1)$.

To prove this corollary, we simply note that $Z(\omega, g \circ f)=\omega(\operatorname{det}(g)) Z(\omega, f)$ and then compare the residues of $Z(\omega, g \circ f)$ and $\omega(\operatorname{det}(g)) Z(\omega, f)$ at $\omega=\tilde{\omega}^{-1} \omega_{1 / 3}$ and $\omega=1$. Thus $\Sigma_{1}^{(i, 3)}\left(f, \tilde{\omega}, \frac{2}{3}\right)$ and $\Sigma_{2}(f,-1)$ are indeed invariant distributions as advertised at the end of $\S 3.5$. 
The second consequence of Theorem 3.2 is the functional equation for $Z(\omega, f)$. Set $I^{-}(\omega, f)=I(\omega, f)-\frac{1}{2} I^{0}(\omega, f)$. Then the explicit formula for $I^{-}(\omega, f)$ of Theorem 3.2 shows that $I^{-}\left(\omega_{2} \omega^{-1}, \hat{f}\right)=I^{0}(\omega, f)+I^{-}(\omega, f)$. By Corollary 3.8.1, $I^{0}\left(\omega_{2} \omega^{-1}, \hat{f}\right)=I^{0}(\omega, f)$, and therefore $I\left(\omega_{2} \omega^{-1}, \hat{f}\right)=I(\omega, f)$. Hence the following corollary.

COROLlary 3.2.3. The zeta function $Z(\omega, f)$ satisfies the functional equation

$$
Z\left(\omega_{2} \omega^{-1}, f\right)=Z(\omega, f) .
$$

This corollary concludes our discussion of the global properties of the adelic zeta function.

\section{BIBLIOGRAPHY}

1. F. Arndt, Zur Theorie der binaren kubischen Formen, J. Reine Angew. Math. 53 (1857), 309-321.

2. A. Borel, Some finiteness properties of adele groups over number fields, Publ. Math. I.H.E.S. 16 (1963), $101-126$.

3. B. Datskovsky, On zeta functions associated with the space of binary cubic forms with coefficients in a function field, Ph.D. thesis, Harvard Univ., 1984.

4. J. Cogdell, Congruence zeta functions for $M_{2}(\mathbf{Q})$ and their associated modular forms, Math. Ann. 266 (1983), 141-198.

5. H. Davenport, On the class-number of binary cubic forms. I and II, J. London Math. Soc. 26 (1951), 183-198.

6. H. Davenport and H. Heilbronn, On the density of discriminants of cubic fields. I, Bull. London Math. Soc. 1 (1969), 345-348.

7. On the density of discriminants of cubic fields. II, Proc. Roy. Soc. London Ser. A 322 (1971), 405-420.

8. B. Delone and D. Faddeev, The theory of irrationalities of the third degree, Amer. Math. Soc. Transl. 10 (1964).

9. G. Eisenstein, Untersuchungen über die cubischen Formen mit zwei Variabeln, J. Reine Angew. Math. 27 (1844), 89-104.

10. G. Harder, Minkowskische Reduktionstheorie über Funktionenkörpern, Invent. Math. 7 (1969), $33-54$.

11. S. Gelbart, Automorphic forms on adele groups, Princeton Univ. Press, Princeton, N. J., 1975.

12. R. Godement and H. Jacquet, Zeta functions of simple algebras, Lecture Notes in Math., vol. 250 , Springer-Verlag, Berlin and New York, 1972.

13. J.-I. Igusa, On certain representations of semi-simple algebraic groups and the arithmetic of the corresponding invariants. I, Invent. Math. 12 (1971), 62-94.

14. New York, 1978.

15. Some results on p-adic complex powers, Amer. J. Math. 106 (1984), 1013-1032.

16. T. Kubota, Elementary theory of Eisenstein series, Wiley, New York, 1973.

17. R. Langlands, Eisenstein series, Proc. Sympos. Pure Math., vol. 9, Amer. Math. Soc., Providence, R. I., 1966, pp. 235-252.

18. On the functional equations satisfied by Eisenstein series, Lecture Notes in Math., vol. 544 , Springer-Verlag, Berlin and New York, 1976.

19. W. Li, On modular functions in characteristic p, Trans. Amer. Math. Soc. 246 (1978), 231-259.

20. , Eisenstein series and decomposition theory over function fields, Math. Ann. 240 (1979), $115-139$.

21. M. Muro, Microlocal analysis and the calculations of functional equations and residues of zeta functions associated with the vector spaces of quadratic forms (preprint).

22. F. Sato, Zeta functions in several variables associated with prehomogeneous vector spaces I: Functional equations, Tôhoku Math. J. (2) 34 (1982), 437-483.

23. M. Sato, M. Kashiwara, T. Kimura and T. Oshima, Microlocal analysis on prehomogeneous vector spaces, Invent. Math. 62 (1980), 117-179. 
24. M. Sato and T. Shintani, On zeta functions associated with prehomogeneous vector spaces, Ann. of Math. (2) 100 (1974), 131-170.

25. T. Shintani, On Dirichlet series whose coefficients are class-numbers of integral binary cubic forms, J. Math. Soc. Japan 24 (1972), 132-188.

26. On zeta-functions associated with the vector space of quadratic forms, J. Fac. Sci. Univ. Tokyo Sect. Ia Math. 22 (1975), 25-66.

27. T. Suzuki, On zeta functions associated with quadratic forms with variable coefficients, Nagoya Math. J. 73 (1979), 117-147.

28. J. Tate, Fourier analysis in number fields and Hecke's zeta function, Ph.D. thesis, Princeton Univ., 1950 .

29. A. Weil, Adeles and algebraic groups, Birkhäuser, Basel, 1982.

30. __ Sur certains groupes d'opérateurs unitaires, Acta Math. 111 (1964), 143-211.

31. __ Sur la formule de Siegel dans la théorie des groupes classiques, Acta Math. 113 (1965), 1-87.

32. __ Fonction zêta et distributions, Séminaire Bourbaki, No. 316, 1966; Math. Ann. 168 (1967), $140-156$.

33. __ Basic number theory, Springer-Verlag, Berlin and New York, 1974.

34. On the analogue of the modular group in characteristic $p$, Proc. Conf. in honor of M. Stone, Springer-Verlag, Berlin and New York, 1970, pp. 211-223.

35. D. Wright, Dirichlet series associated with the space of binary cubic forms with coefficients in a number field, Ph.D. Thesis, Harvard Univ., 1982.

36. D. J. Wright, The adelic zeta function associated with the space of binary cubic forms, I: Global theory, Math. Ann. 270 (1985), 503-535.

Department of Mathematics, Tufts University, Medford, Massachusetts 02155

Current address: Department of Mathematics, Harvard University, Cambridge, Massachusetts 02138 